\title{
Roles of texture and latent hardening on plastic anisotropy of face-centered-cubic materials during multi-axial loading
}

M.S. Pham ${ }^{1,2^{*}}$, A. Creuziger ${ }^{2}$, M. Iadicola ${ }^{2}$ and A.D. Rollett ${ }^{1}$

${ }^{1}$ Materials Science and Engineering, Carnegie Mellon University, 5000 Forbes Avenue, Pittsburgh, PA 15213, USA

${ }^{2}$ NIST Center for Automotive Lightweighting Center, National Institute of Standards and Technology, 100 Bureau Drive, Gaithersburg, MD 20899-8553, USA

Abstract: This study investigates the joint impact of preferred texture and latent hardening on the plastic anisotropy of face centered cubic (FCC) materials. The main result is that both aspects have significant impact on the anisotropy, but the two can either counteract each other or synergistically reinforce each other to maximize anisotropy. Preferred texture results in significant anisotropy in plastic yielding. However, the latent hardening significantly alters the texture-induced anisotropy. In addition, one latent hardening type can cancel out the anisotropy of another type. Consequently, if all dislocation-based latent hardening types are included at the same level as the self-hardening, the result might not reveal the complexity of plastic anisotropy. The present study of the synergistic influence of detailed latent hardening and texture presented helps provide new insights into the complex anisotropic behavior of FCC materials during multiaxial forming. 


\section{Terminology:}
AR
As-received
$b$
slip direction
EB
Equal biaxial
CRSS
Critical resolved shear stress
COL
Collinear
COP
Co-planar
FCC
Face-centered cubic
GL
Glissile
$\mathrm{H}$
Hirth
$h_{\alpha \beta}$
Hardening coefficient induced by the interaction between dislocations on the slip systems and $\beta$
LC
Lomer-Cottrell
$m^{\alpha}$
Schmid factor of the slip system
$N$
Number of slip systems
$n$
Slip plane normal
$n^{\text {eff }}$
PS-RD
$n$-effective parameter represents the interaction between the ellipsoidal inclusion and matrix in VPSC
Plane strain with the maximum strain in the rolling direction and zero strain in the transverse direction
PS-TD
Plane strain with the maximum strain in the transverse direction and zero strain in the rolling direction
RD
Rolling direction of sheet material
$S$
Shear texture
TD
Direction orthogonal (transverse) to the sheet rolling direction
U-RD, U-TD
Uniaxial straining along the RD direction, the TD direction, respectively
VPSC
Visco-plastic self-consistent
$\alpha, \beta$
Slip systems
Shear rate
$\sigma_{0}$
Initial yield strength 
$\sigma_{R D}, \sigma_{T D}$

$\tau^{\alpha}$

$\tau_{0}, \tau_{1}$

$\theta_{0}, \theta_{1}$
True stress in the RD direction and in the TD direction

Resolved shear stress of applied stress on the slip system

Critical shear stress at the beginning and at the large deformation stage of plastic deformation

Hardening rates at the beginning and at the large deformation stages of plastic deformation 


\section{Introduction}

Automotive part production demands accurate modeling and simulation of the material response during metal forming processes. It is straightforward to predict the isotropic behavior (a material behaves identically in all loading directions). The von Mises hypothesis is one of the most common approaches for predicting the isotropic response at the continuum scale. The von Mises hypothesis of isotropy assumes that the flow stress in the deviatoric stress space at the continuum scale should follow these conditions:

(1) the stress at which the material initially yields is not a function of material orientation with respect to the frame of the test (i.e., isotropic yielding);

(2) there exists a multi-axial yield locus that is described by a single value of stress that corresponds to yield in uniaxial tension (i.e., stress equivalency);

(3) on hardening, the multi-axial yield locus expands by the same amount in every direction in the $\pi$-plane, which is the plane that has its normal parallel to [111] in the deviatoric stress space (i.e., isotropic hardening);

(4) there is an associated flow rule, i.e., the strain increment is normal to the yield locus.

Requirements (3) and (4) lead to a consequence that a stress ratio corresponding to any given strain ratio remains constant and is equal to the strain ratio during plastic deformation. In other words, there are specific stress (or strain) ratios for different loading paths. In this present study, "loading" can be either mechanical straining or stressing, and a loading path means a locus of stress (or strain) states that a material is subjected to during deformation. Examples of (monotonic) loading paths in this study include uniaxial tension, plane strain tension and balanced biaxial tension. In addition, isotropic hardening requires that the hardening rate is the same for all loading paths.

In practice, it is challenging to accurately model complex behavior during multi-axial forming along various loading paths because most engineering materials behave differently along different loading directions, i.e., anisotropic behavior. The anisotropic behavior can be considered as the deviation of material response from an ideally isotropic response (e.g., von Mises isotropy). In this paper, the plastic anisotropy will be represented by the deviation in both the initial plastic yield and hardening behavior, while the anisotropic hardening is characterized by the evolution of stress (or strain) and the increment in stress (i.e., hardening rate) during plastic deformation. It is often seen that stress (or strain) paths, in response to uniaxial (or plane strain or equi-biaxial) deformation, do not follow ideal isotropic stress (or strain) paths. The hardening rate is also not the same for all loading paths. During forming, the anisotropic 
response of engineering materials during plastic deformation is complex and challenging to model accurately. This study aims to understand the plastic anisotropic response during multiaxial loading of face-centered-cubic materials via a dislocation-based approach in order to provide information for improving constitutive models for accurate predictions of the material behavior during forming.

There has been substantial modeling effort devoted to progressing from empirical models to physics-based models, particularly for modeling anisotropy [1-3], because these approaches are expected to enhance the accuracy and effectiveness of models. The anisotropy is known to be a function of the crystallographic texture of the material [4]. In addition, it is also known that dislocation behaviors, such as dislocation interactions (both with other dislocations and other defects), result in changes in the yielding and hardening behavior [5-8]. One of most obvious manifestations of plastic anisotropy due to dislocations is referred to as latent hardening (also known as "cross-hardening"). Latent hardening is used to describe yielding and hardening phenomena, where a higher yield strength and higher hardening rate are often observed in polycrystalline materials when the strain path changes $[5,8]$. Latent hardening results from the fact that the activity on one slip system affects other systems to different degrees because the stored dislocations on one system act as forest dislocations on intersecting slip systems [5, 8]. In particular, during sheet metal forming, the multiaxial stress state induces slip activity on multiple systems, resulting in the formation of a spectrum of dislocation junctions and their associated strengths [5, 8-10]. The junctions act as obstacles to mobile dislocations on intersecting slip systems. Strain path changes can activate new sets of dislocation interactions, resulting in different dislocation junctions and, consequently, changes in hardening behavior [5, 8, 11]. Even during monotonic loading, a specific loading path results in the formation of dislocation junction types different than those along other paths, leading to anisotropic hardening behavior for different monotonic loading paths $[5,8]$. In other words, dislocation interaction-based latent hardening significantly contributes to plastic anisotropy not only during loading path changes, but also during different monotonic loading paths. Consequently, to understand and then accurately model the mechanical response during forming, it is necessary to study not only the influence of texture on plastic anisotropy, but also (1) those of dislocation interaction-induced latent hardening and (2) the synergistic influences of both texture and latent hardening.

Although dislocation-based hardening models have been intensively applied in constitutive models for plastic anisotropy, most models employ a self-hardening law based on self- 
interaction of dislocations. Recently, there have been efforts to include non-self-interactions of dislocations in constitutive models to account for latent hardening. For example, clear differentiation between self and non-self-interactions [12-14] (or between coplanar and noncoplanar interactions [15], or between collinear and non-collinear interactions [16]) was used to better account for latent hardening. There have also been efforts to include more detailed dislocation interactions (e.g., [9, 17-20]). However, it still requires significant effort to understand the plastic anisotropy under the influence of specific latent hardening sources and texture conditions, in particular during multi-axial forming conditions. It is also interesting to note that it is unclear whether latent hardening influences anisotropy and/or texture evolution. Kocks et al. showed that latent hardening has little effect on texture development, as well as on the plastic anisotropy (except for compression) [6]. However, more recent work argued that latent hardening can significantly affect the evolution of texture compared to self-hardening $[15,21$, 22]. Consequently, the present study aims at understanding the plastic anisotropy under the presence of texture with and without latent hardening to provide some insight for the abovementioned discrepancies in the literature.

In order to explicitly account for dislocation interactions, one could use discrete dislocation dynamics, which, however, are currently limited to single crystals or idealized multi-crystal domains. When one realizes that the problems in metal forming involve complex polycrystals, it is not practical to use discrete dislocation dynamics to systematically study the anisotropy of materials in multi-axial deformation along complex load paths with various initial textures. Our objective is to use a simulation platform that enables a reasonable prediction of texture evolution of an ensemble of grains during deformation while allowing for the inclusion of dislocation interactions inside each grain. This enables us to study the separate and joint effects of textures and latent hardening on the anisotropy of metals. The self-consistent approach is known for its texture prediction capability with the large number of grains [3] that is necessary for sheet metal forming while being only moderately computationally expensive. It is readily adapted to include effects of latent hardening [20,23]. In this study, all types of dislocation interactions in pairs in face-centered-cubic (FCC) materials and texture are incorporated in a visco-plastic self-consistent (VPSC) model to understand the relationships between latent hardening, texture and plastic anisotropy. The plastic anisotropy of FCC materials as a function of typical textures along a number of monotonic straining paths with self-hardening is first investigated as a baseline (Sect. III.3). Corresponding discussions of these results are given in Sect. IV.2. Subsequently, the effects of dislocation-based latent hardening without the presence of preferred texture on plastic anisotropy are presented in Sect. III.4 and discussed in Sect. IV.3. 
Finally, plastic anisotropy under the presence of both preferred textures and latent hardening is investigated to understand the synergistic influences of texture and latent hardening on the overall anisotropic response (Sects. III.5-9 and IV.4). Based on this analysis, we seek possible explanations (Sect. IV.4) for the discrepancy in the influence of latent hardening on plastic anisotropy between Kocks and other studies as referred to above [6, 15, 21].

\section{Modeling}

\section{II.1. Stress-strain constitutive model}

The stress-strain constitutive relationship is described in this section. The detailed treatment of the deformation slip kinematics is given in [24]. At the grain level, the plastic strain rate $\dot{\varepsilon}^{p}$ is given by the sum of the shear strain rates $\dot{\gamma}^{\alpha}$ from all the active slip systems

$$
\dot{\varepsilon}^{p}=\sum_{\alpha=1}^{N} m^{\alpha} \dot{\gamma}^{\alpha}
$$

where $N$ is the number of slip systems, $m^{\alpha}$ is the Schmid tensor of the slip system. The Schmid tensor is defined as

$$
m^{\alpha}=\frac{1}{2}\left(b^{\alpha} \otimes n^{\alpha}+n^{\alpha} \otimes b^{\alpha}\right)
$$

where $b$ is the slip direction and $n$ is the slip plane normal. The rate at which dislocations move under the influence of a shear stress on their glide plane depends on the magnitude of the shear stress. This rate sensitivity can be modeled as a power-law relationship between the Schmid resolved shear stress and the shear rate $\dot{\gamma}^{\alpha}$ on slip system $\alpha[25,26]$,

$$
\dot{\gamma}^{\alpha}=\dot{\gamma}_{0}^{\alpha}\left|\frac{\tau^{\alpha}}{\hat{\tau}^{\alpha}}\right|^{\mathrm{r}} \operatorname{sgn}\left(\tau^{\alpha}\right)
$$

with $\dot{\gamma}_{0}^{\alpha}$, and $\wedge$ are respectively defined as the reference shear rate, resolved shear stress, and the critical resolved shear stress for dislocation motion in the slip system $\alpha$. The inversion of the rate sensitivity exponent $(r)$ is assumed to be constant during isothermal deformation process and to be equal for all slip systems. The value of $r$ is in the range of 1/0.03 at room temperature to $1 / 0.3$ at elevated temperatures according to Kocks [27]. Therefore, the value $r=20$ used in our previous study [28] was also used in this study. 
The critical resolved shear stress (CRSS) of the slip system $\left.\alpha{ }^{\wedge}{ }^{\wedge}\right)$ evolves during plastic deformation as a function of accumulated plastic strain thanks to the motion of dislocations in $\alpha$. The ${ }^{\wedge}$ is calculated by an extended Voce-type model $([24,29])$ as follows,

$$
\hat{\tau}^{\alpha}=\hat{\tau}_{0}^{\alpha}+\left(\hat{\tau}_{1}^{\alpha}+\hat{\theta}_{1}^{\alpha} \gamma\right)\left(1-\exp \left(-\gamma \frac{\hat{\theta}_{0}^{\alpha}}{\hat{\tau}_{1}^{\alpha}}\right)\right)
$$

where, $\hat{\tau}_{0}^{\alpha}, \theta_{0}^{\alpha}$, and $\theta_{1}^{\alpha}$ are the initial critical resolved shear stress, the initial hardening and the asymptotic hardening rates. $\hat{\tau}_{0}^{\alpha}+\hat{\tau}_{1}^{\alpha}$ is the back-extrapolated critical resolved shear stress that is the $y$-intercept of a line that has the slope of $\theta_{1}^{\alpha}$ and is tangential to the $\hat{\tau}^{\alpha}=\hat{\tau}^{\alpha}(\gamma)$ at large strain, $\gamma$ is the total accumulated shear plastic strain in a grain. Although the original Voce model [30] was based on a phenomenological fit to experimental data, the extended Voce model was developed on the basis of the understanding of the dislocation density evolution proposed by Kocks and Mecking [31, 32]. Kocks showed that the extended Voce model indeed reflects the hardening behaviour Stage II of FCC materials [32]. One should note that Stage II is governed by the interaction of dislocations (Section 2.5 in [33]), which is the topic of the present study and has demonstrated to be very effective in capturing the hardening behavior of a wide range of metals [34-38]. In addition, the way the extended Voce model relates latent hardening to dislocation interactions (refer to Sects. II.2 and II.3) is similar to what was proposed by Franciosi [8]. This treatment of latent hardening enables the linkage between microscopic interactions of dislocations with macroscopic quantities at large scales.

In this study, we utilized a VPSC program (version 7b) that was intensively developed at the Los Alamos National Laboratory [3]. Parameters for the Voce-type constitutive model (Eqn. 4) were identified by fitting experimental stress-strain data in the rolling direction during equi-biaxial (EB) straining of an aluminum alloy (AA) 5754-O as described in [11]. The identified parameters (given in Tab. 1) are slightly different from those given in [11] because the latter were optimized against both stress-strain data and texture development after $20 \%$ EB straining. The grainmatrix interaction used in this study is in between the stiff secant (which tends to a uniform strain state across grains, i.e., Taylor assumption) and the compliant tangent (the lowest bound of this approximation is a uniform stress state, i.e., Sachs hypothesis). This study used the $\mathrm{n}^{\text {eff }}$ adjustable parameter (of grain-matrix interaction [24]) of 10 that was found to work well with 
FCC materials (e.g., aluminum alloys [39]). The parameters given in Table 1 were used to simulate plastic anisotropy in response to five loading paths of different initial texture conditions. Table 1: Voce hardening parameters for VPSC

\begin{tabular}{ccccc}
\hline Grain-Matrix interaction & $\begin{array}{c}\hat{\tau}_{0} \\
(\mathrm{MPa})\end{array}$ & $\begin{array}{c}\hat{\tau}_{1} \\
(\mathrm{MPa})\end{array}$ & $\begin{array}{c}\theta_{0} \\
(\mathrm{MPa})\end{array}$ & $\begin{array}{c}\theta_{1} \\
(\mathrm{MPa})\end{array}$ \\
\hline$n^{\text {eff }}=10$ & 81 & 24 & 80 & 0 \\
\hline
\end{tabular}

\section{II.2. Self and Latent hardening models}

The interactions between slip systems $\alpha$ and $\beta$ during plastic deformation result in a change in the CRSS ( ${ }^{\wedge}$ ) of slip system $\alpha$. The evolution of ${ }^{\wedge}$ can be calculated by the following equation [24]:

$$
d \hat{\tau}^{\alpha}=\frac{d \hat{\tau}^{\alpha}}{d \gamma} \sum_{\beta=1}^{N} h_{\alpha \beta} d \gamma^{\beta} \quad(\alpha \text { and } \beta=\overline{1, N})
$$

where, $\frac{d \hat{\tau}^{\alpha}}{d \gamma}=\theta_{1}^{\alpha}+\left(\theta_{0}^{\alpha}-\theta_{1}^{\alpha}\right) \exp \left(-\gamma \frac{\theta_{0}^{\alpha}}{\hat{\tau}_{1}^{\alpha}}\right)+\theta_{1}^{\alpha} \frac{\theta_{0}^{\alpha}}{\hat{\tau}_{1}^{\alpha}} \gamma \exp \left(-\gamma \frac{\theta_{0}^{\alpha}}{\hat{\tau}_{1}^{\alpha}}\right)$ is the change in the CRSS with respect to the change in $\gamma, \gamma=\sum_{\alpha=1}^{N}\left|\gamma^{\alpha}\right|$ is the accumulated plastic strain in the grain, and $h_{\alpha \beta}$ is the hardening matrix associated with the contributions of the slip activity of slips $\beta$ to the increment of the CRSS of slip $\alpha$ due to dislocation interactions between slip systems $\alpha$ and $\beta$. Depending on the type of dislocation interactions between slip systems $\alpha$ and $\beta, h_{\alpha \beta}$ represents the strength of corresponding dislocation interactions relative to that of $h_{\alpha \alpha}$ (the self-interaction). The $h_{\alpha \alpha}$ is set equal to 1 and used as the reference. For the self-hardening, all components $h_{\alpha \beta}$ of the hardening matrix are equal to 1. In other words, (1) every slip system hardens based on any slip systems being active and (2) all dislocation interactions between active slip systems are considered to be the same as the self-interactions. For the case of latent hardening, it is expected that dislocation junctions associated with non self-interactions should have strength stronger than that of the self-hardening [8]. Therefore, $h_{\alpha \beta}$ should be greater than (or equal to) 1 . 


\section{II.3. Dislocation interactions and latent hardening in FCC materials}

If there is no differentiation in the sign of slip directions, there are twelve $\{111\}<110>$ slip systems for dislocation movements in FCC materials, i.e., $N=12$ in Eqn. 5. The slip systems in FCC materials are depicted by the Thompson tetrahedron in Fig. 1 [40]. In addition to dislocation self-interactions (i.e., interactions of dislocations moving in the same slip system $\alpha$ ), there are five types of interactions between dislocations in different slip systems (Table 2). The most frequent interactions are Lomer-Cottrell (LC) interactions, which result in the formation of LC junctions [41-43]. The other dislocation interactions are Hirth $(\mathrm{H})$, coplanar (COP), glissile (GL) and collinear (COL) [10, 44, 45]. Different types of dislocation junctions have different strengths, so do the dislocation interaction types, leading to different hardening rates. The nonself-interactions usually induce higher hardening rates than that induced by self-interaction, i.e., latent hardening causes higher hardening rates than the self-hardening [5-8]. Hereinafter, latent hardening resulting from $\mathrm{COL}, \mathrm{COP}, \mathrm{GL}, \mathrm{H}$ or $\mathrm{LC}$ interactions is defined as COL, COP, GL, H, or LC latent hardening, respectively. Because $\mathrm{COP}$ and $\mathrm{GL}$ dislocation interactions share the same slip direction and are usually treated as being the same [44], COP and GL are also grouped together in this study.

\section{(a) Thompson tetrahedron}

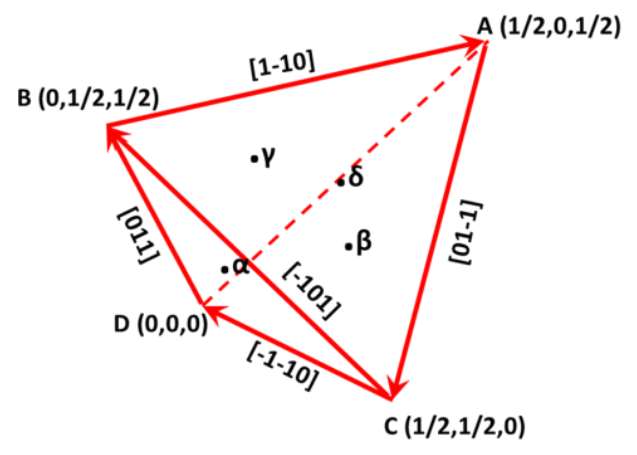

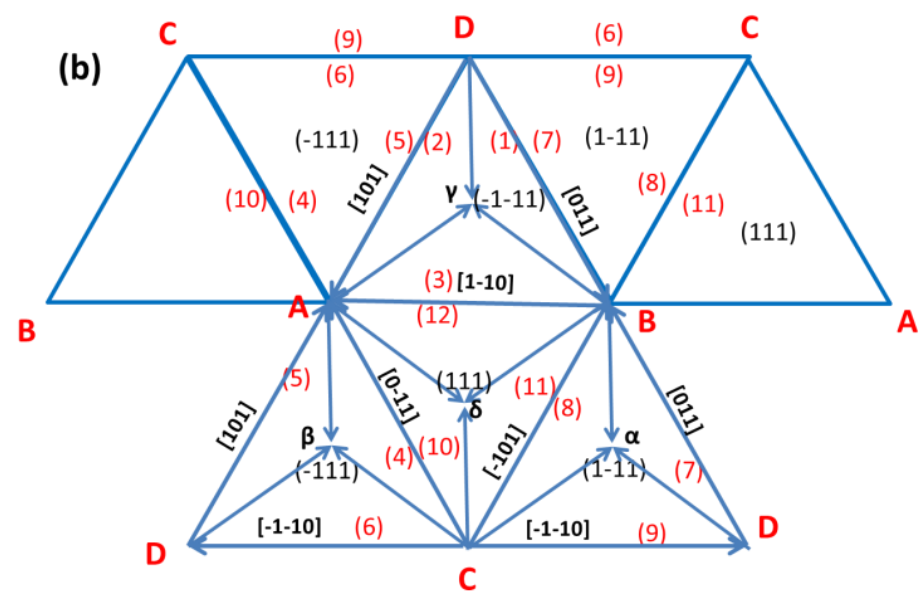

Figure 1: (a) Thompson tetrahedron after [40] and (b) its expansion to describe slip systems (shown in parentheses) in FCC materials. 
Table 2: Dislocation interactions in FCC materials (note that the matrix is not symmetric); refer to Figure $1 \mathrm{~b}$ for detailed description of slip system numbers.

\begin{tabular}{cccccccccccccc}
\hline$a$ & 1 & 2 & 3 & 4 & 5 & 6 & 7 & 8 & 9 & 10 & 11 & 12 \\
\hline 1 & Self & COP & COP & H & GL & LC & COL & LC & LC & H & LC & GL \\
\hline 2 & COP & Self & COP & LC & COL & LC & GL & H & LC & LC & H & GL \\
\hline 3 & COP & COP & Self & LC & GL & H & GL & LC & H & LC & LC & COL \\
\hline 4 & H & GL & LC & Self & COP & COP & H & LC & GL & COL & LC & LC \\
\hline 5 & LC & COL & LC & COP & Self & COP & LC & H & GL & GL & H & LC \\
\hline 6 & LC & GL & H & COP & COP & Self & LC & LC & COL & GL & LC & H \\
\hline 7 & COL & LC & LC & H & LC & GL & Self & COP & COP & H & GL & LC \\
\hline 8 & GL & H & LC & LC & H & GL & COP & Self & COP & LC & COL & LC \\
\hline 9 & GL & LC & H & LC & LC & COL & COP & COP & Self & LC & GL & H \\
\hline 10 & H & LC & GL & COL & LC & LC & H & GL & LC & Self & COP & COP \\
\hline 11 & LC & H & GL & GL & H & LC & LC & COL & LC & COP & Self & COP \\
\hline 12 & LC & LC & COL & GL & LC & H & LC & GL & H & COP & COP & Self \\
\hline
\end{tabular}

There is a $12 \times 12$ hardening $h_{\alpha \beta}$ matrix that corresponds to the $12 \times 12$ matrix of dislocation interactions (Table 2). The types of dislocation interactions in Table 2 are included in the Vocetype model (Eqn. 4) through the corresponding $h_{\alpha \beta}$. As mentioned above, it is expected that dislocation junctions associated with non self-interactions should have a strength higher than that of self-hardening [5-8]. In this study, the hardening coefficient of a type of dislocation interactions is set to be 2.5, $h_{\alpha \beta}=2.5$ for $\alpha \neq \beta$. The value of 2.5 is in the middle of the range of latent hardening reported in [8]. To study the influence of latent hardening associated with a type of dislocation interactions between $\alpha$ and $\beta$ on the plastic anisotropy, only $h_{\alpha \beta}$ components corresponding to the considered type of dislocation interactions (as shown in Tab. 2) are assigned the value of 2.5 (while the rest of the components associated with other interaction types are set to be the same as the self-hardening). For example, the "COL" collinear latent 
hardening means that only the components $h_{\alpha \beta}$ corresponding to COL interactions in Table 2 are set to equal to 2.5 while other $h_{\alpha \beta}$ components associated with other interaction types (i.e., COP, GL, H, LC and self) are equal to 1 . This process is done for each type of latent hardening. The plastic anisotropic behavior of FCC materials, when all latent hardening sources are active and have the same strength, was also investigated. This is equivalent to the case where only self- and non-self-interactions of dislocations are differentiated, which was also often used in previous studies $[12,22]$.

\section{II.4. Initial crystallographic textures}

Various initial crystallographic textures were used to study the separate influence of texture, and the coupling effects of texture and latent hardening on the anisotropy. In this paper, sets of 2,000 orientations near an ideal texture component (either Uniform (Random), Cube, Copper, Brass, S, or Goss (G), Fig. 2, Tab. 3) were generated by MTEX (version 3.4.1 [46]) and used as the input texture file for VPSC calculations. For each component (except for the Random texture), the half width was $5^{\circ}$, and cubic crystal symmetry and orthotropic (orthorhombic) sample symmetry were applied. Figure 2 depicts five texture components, as well as two fibers often seen in FCC materials, i.e., $\alpha$-fiber (running from Goss to Brass components) and B-fiber (connecting the Brass, $\mathrm{S}$ and Copper components). For each initial texture and a single hardening type (either self-hardening, $\mathrm{COL}, \mathrm{COP}=\mathrm{GL}, \mathrm{H}$, or $\mathrm{LC}$ latent hardening types), a set of five simulations of the flow stress and texture development during five linear loading paths (e.g., two uniaxial, two plane strain and one equal biaxial paths) was carried out. Detailed boundary conditions for these loading paths, in terms of the velocity gradient tensor, are described in Sect. II.5 and summarized in Table 4. 


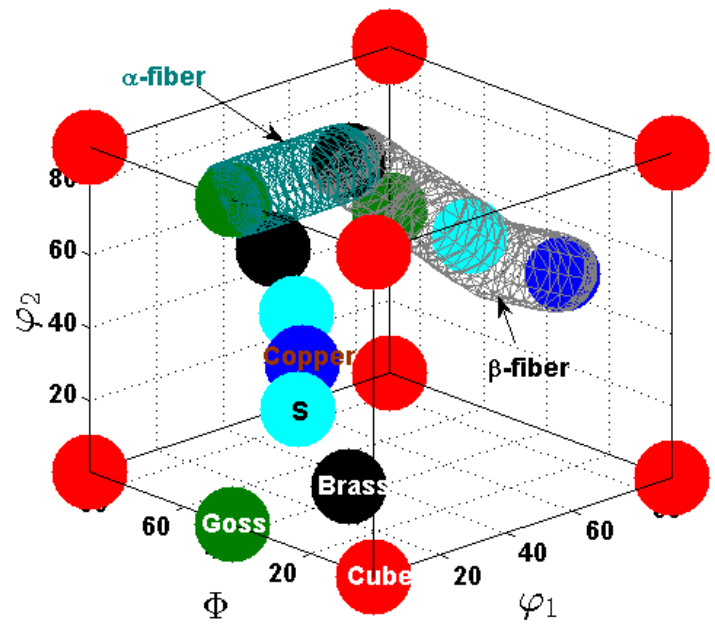

Figure 2: Typical textures of FCC materials shown in the Bunge convention.

Table 3: Individual texture components specified in Bunge Euler angle notation.

\begin{tabular}{|c|c|c|c|c|}
\hline \multirow{2}{*}{ Texture } & \multirow{2}{*}{ Miller indices } & \multicolumn{3}{|c|}{$\left(\varphi_{1}, \theta, \varphi_{2}\right)$} \\
\hline & & Variant 1 & Variant 2 & Variant 3 \\
\hline Cube $^{(1)}$ & $\{001\}\langle 100\rangle$ & $(0,0,0)$ & & \\
\hline Copper & $\{112\}\langle 11 \overline{1}\rangle$ & $(40,65,26)$ & $(90,35,45)$ & \\
\hline S & $\{123\}\langle 63 \overline{4}\rangle$ & $(32,58,18)$ & $(48,75,34)$ & $(64,37,63)$ \\
\hline Brass & $\{110\}\langle\overline{11} 2\rangle$ & $(35,45,0)$ & $(55,90,45)$ & $(35,45,90)$ \\
\hline Goss & $\{110\}\langle 001\rangle$ & $(0,45,0)$ & $(90,90,45)$ & $(0,45,90)$ \\
\hline
\end{tabular}

Note: ${ }^{(1)}$ Only one of the many symmetry-related variants of the Cube component is listed because of their large number. The $90 \times 90 \times 90^{\circ}$ sub-volume of orientation space contains three copies of the fundamental zone for cubic-orthrhombic symmetry. 


\section{II.5. Loading paths}

Uniaxial (U), plane strain (PS), and equal biaxial (EB) strain paths of sheet metals were considered in this study. Detailed boundary conditions for the PS and EB strain paths, in terms of the velocity gradient tensor, are given in Table 3 . The loading conditions for uniaxial strain paths were done by imposing both one normal velocity gradient component (e.g., RD component for uniaxial in RD) and other normal stress components (e.g., TD and ND for uniaxial in RD) in such a way to assure uniaxial stress states (i.e., $\sigma_{T D}=\sigma_{N D}=0$ or $\sigma_{R D}=\sigma_{N D}=0$, note: ND is the normal direction to sheet metal) for uniaxial straining in the rolling direction (RD) or the transverse direction (TD), respectively.

Table 3: Boundary condition for linear straining path

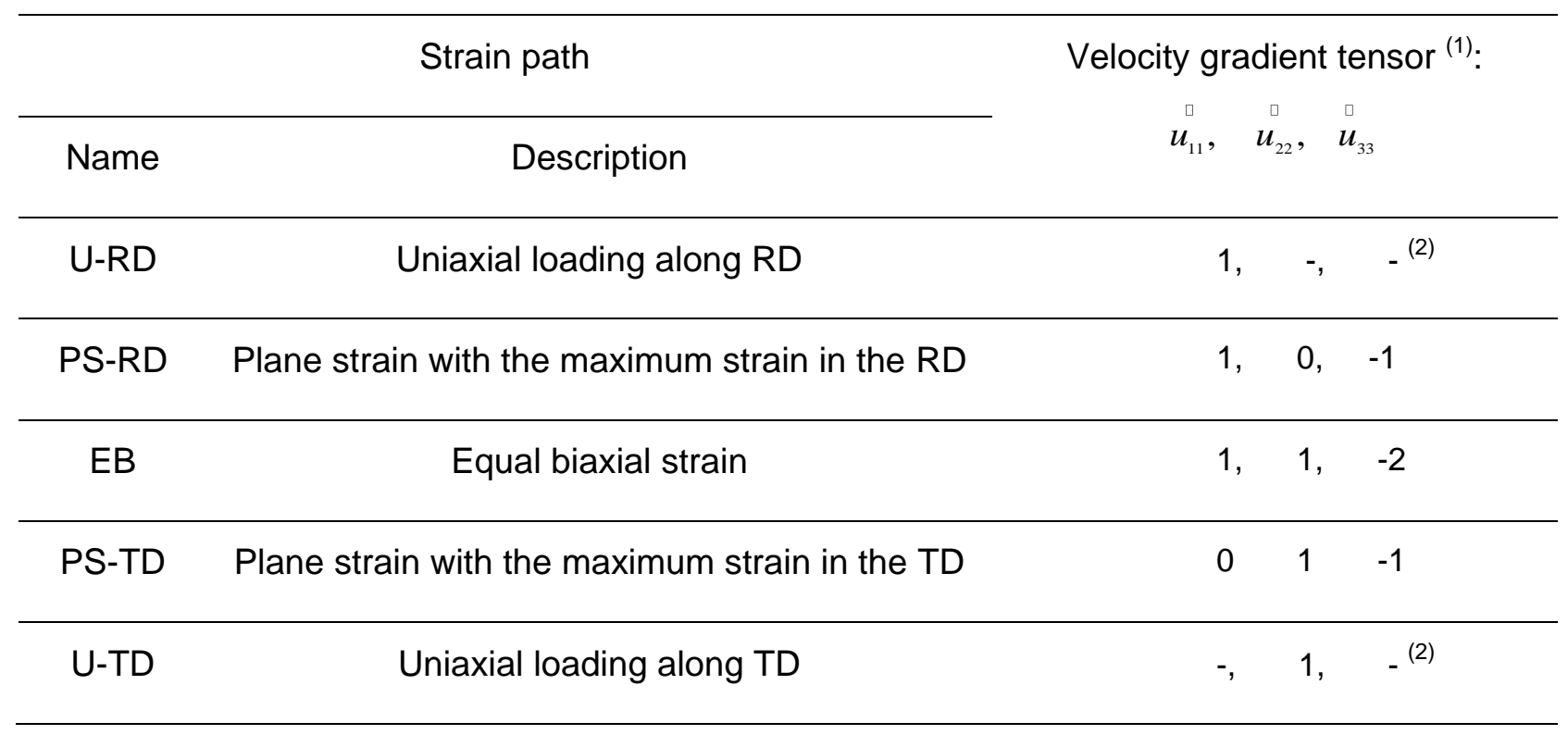

Note: ${ }^{(1)}$ other tensor components equal to zero. ${ }^{(2)}$ see text for details of the applied uniaxial stress state.

\section{II.6. Data post-processing}

In-plane straining should result in plane stress conditions (i.e., the stress component $\sigma_{N D}$ normal to metal sheets is zero). However, the values of all simulated stress components given in the VPSC output are deviatoric stresses that result in a non-zero and negative third deviatoric stress component $\left(\sigma_{33}^{\prime}\right)$ in the VPSC output. In order to comply with the in-plane stress condition,the absolute value of the third normal deviatoric stress $\left(\sigma_{33}^{\prime}\right)$ was added to all three output stress components on the leading diagonal in order to assure $\sigma_{N D}=0$ and provide 
Cauchy stresses comparable to the uniaxial tests. The stress paths were then plotted in 2-D stress coordinates $\left(\sigma_{R D}, \sigma_{T D}\right)$ such that the plastic work done of a deformation step during loading along a certain path is equal to that done after $1 \%$ strain increment of U-RD. The plastic work for incompressible materials is defined by:

$W=\int\left(\sigma_{i j}-\frac{1}{3} \sigma_{k k} \delta_{i j}\right) d \varepsilon_{i j}^{p}$

Because the RD and TD are also the principal directions, the plastic work was redefined as

$W=\int\left(\sigma_{R D} \varepsilon_{R D}+\sigma_{T D} \varepsilon_{T D}\right)$

For each direction (RD or TD) of a loading condition, a polynomial of degree 4 was used to fit the corresponding stress-strain curve in order to calculate the plastic work.

The texture files required and generated by VPSC include the crystallographic orientations and their associated weights. The weighted lists of orientations were converted to the format of crystal orientation distribution by subroutines wts2cod.f [47]. The crystallographic orientation distribution data was then plotted by in-house MATLAB subroutines [48].
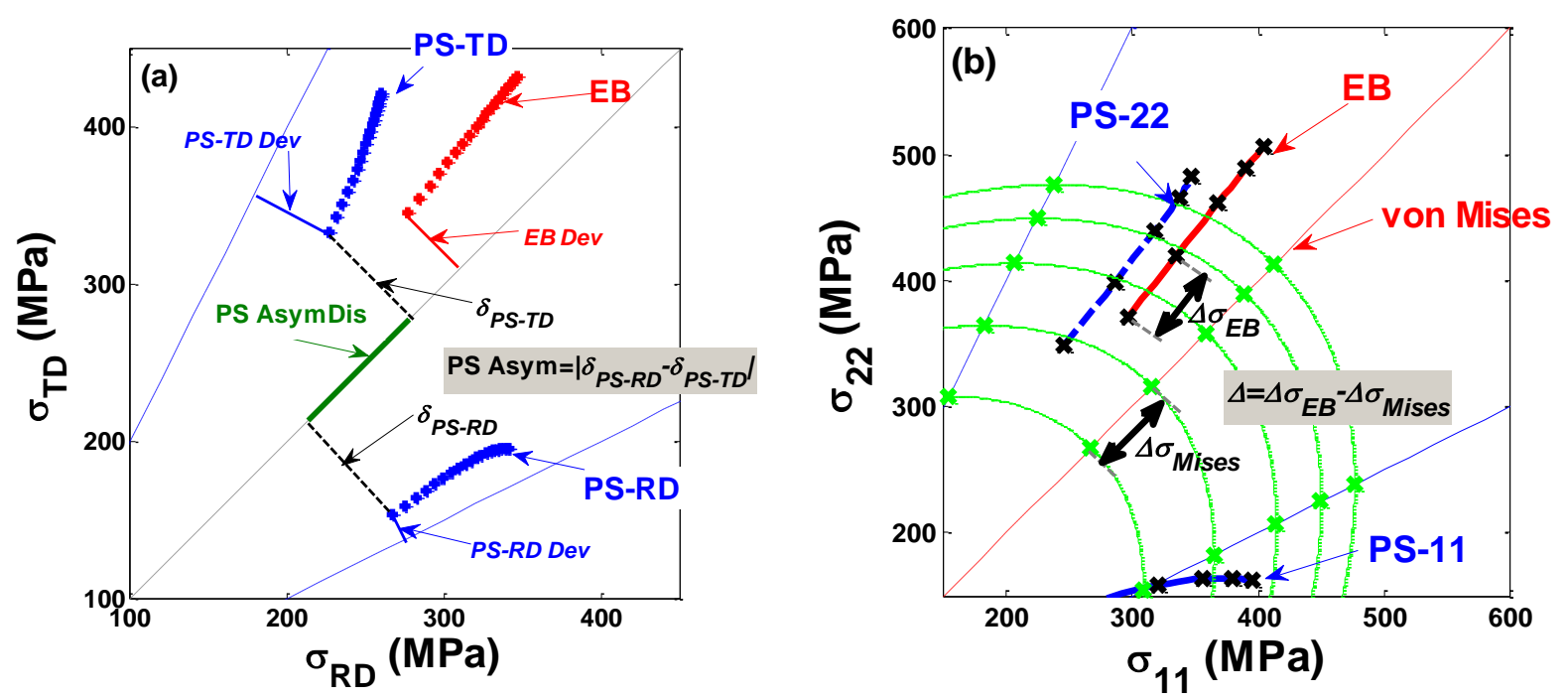

Figure 3: Parameters representing the plastic anisotropy during multi-axial in-plane straining. (a) $P S-R D$ Dev, $P S-T D$ Dev, EBDev : the deviations of PS-RD, PS-TD and EB stress paths from corresponding von Mises isotropic stress lines. (b) $\Delta$ : anisotropic hardening parameter.

Concerning the quantitative representation of plastic anisotropy, four anisotropy parameters are introduced to describe the anisotropic response of stress evolution in the $2 \mathrm{D}$ space. The first 
three parameters quantitatively represent the deviations of stress paths from the corresponding von Mises isotropic stress lines. The deviation of a resulting stress path from the corresponding isotropic line was quantified as the distance from calculated points of the resulting stress during loading up to $20 \%$ to the isotropic line. The isotropic lines corresponding to plane strain along the $\mathrm{RD}$, equi-biaxial strain, and plane strain along the TD are described by $\sigma_{T D}^{i s o P S-R D}=\sigma_{R D}^{i s o P S-R D} / 2$, $\sigma_{T D}^{i s o E B}=\sigma_{R D}^{i s o E B}$ and $\sigma_{T D}^{i s o P S-\mathrm{TD}}=2 \sigma_{R D}^{i s o P S-\mathrm{TD}}$, respectively (Fig.3). The deviations of stress paths in response to PS-RD, EB and PS-TD from the respective isotropic lines are named as the PS-RD Dev, EB Dev and PS-TD Dev, respectively. Fig. 3 depicts how the deviations of stress paths with respect to isotropic lines were calculated. In detail,

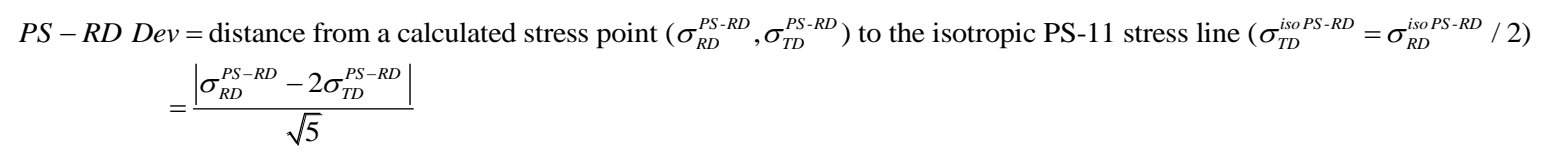

$E B$ Dev $=$ distance from a calculated stress point $\left(\sigma_{R D}^{E B}, \sigma_{T D}^{E B}\right)$ to the isotropic EB stress line $\left(\sigma_{T D}^{i s o E B}=\sigma_{R D}^{i s o E B}\right)$

$$
=\frac{\left|\sigma_{R D}^{E B}-\sigma_{T D}^{E B}\right|}{\sqrt{2}}
$$

$P S-T D D e v=$ distance from a calculated stress point $\left(\sigma_{R D}^{P S-T D}, \sigma_{T D}^{P S-T D}\right)$ to the isotropic PS-TD stress line $\left(\sigma_{T D}^{i s o P S-T D}=2 \sigma_{R D}^{i s o P S-T D}\right)$

$$
=\frac{\left|2 \sigma_{R D}^{P S-T D}-\sigma_{T D}^{P S-T D}\right|}{\sqrt{5}}
$$

In addition, the two PS-RD and PS-TD isotropic stress paths are symmetric about the diagonal line. Therefore, the anisotropy is also manifested by the degree of asymmetry of the PS-RD and PS-TD stress paths about the diagonal line. Other parameters were introduced to represent the asymmetry of the PS-RD and PS-TD stress paths about the diagonal line. In the 2D frame, this asymmetry can be quantified by two parameters. The first one is PS Asym. The PS Asym is equal to $\left|\delta_{P S-R D}-\delta_{P S-T D}\right|:$ the absolute difference between the distances from the PS-RD ( $\left.\delta_{P S-R D}\right)$ and PS-TD ( $\left.\delta_{P S-T D}\right)$ perpendicular to the diagonal line, respectively. The second parameter of asymmetry is the distances between the projection points of the PS-RD stress and PS-TD stress along the diagonal line (refer to PS AsymDis in Fig. 3a).

An additional parameter ( $\Delta$, Fig. $3 b)$ is introduced to quantify the anisotropy in hardening rate. The anisotropic hardening parameter $(\Delta)$ is defined as the difference of stress increment during one deformation step between texture-associated stresses and corresponding von Mises stresses along a specific loading path. For example, the anisotropic hardening parameter for EB stress path is $\Delta=\Delta \sigma_{E B}-\Delta \sigma_{E B}$ von Mises where $\Delta \sigma_{E B}$ is the stress increment of one deformation step 
of texture-associated stresses along the EB stress path, and $\Delta \sigma_{E B \text { vonMises }}$ is the corresponding increment of von Mises stress along the EB von Mises stress path (Fig. 3b).

\section{Results}

The combinations of 5 different paths, 6 hardening models and 6 initial texture components result in 180 different simulations of the flow stress response and texture evolution. In the following sub-sections, we plot stress (or strain) for all 5 different loading paths in a sub-figure. Sub-figures that correspond to the same initial texture are shown in the same row, while those corresponding to a given hardening model are shown in the same column. For plotting the texture development, sub-figures of texture condition of the same initial texture were in the same row. While the first column was for the initial texture, the remaining five columns show the evolved texture after loading along 5 different paths with strain of $20 \%$.

\section{III.1. Strain paths during uniaxial loading}

The conditions for uniaxial loading given in the Table 3 assure a uniaxial stress state, but the strain state may not be uniaxial because of texture-induced anisotropy. Therefore, the strain paths during uniaxial loading in the RD and TD were plotted in Fig. 4. In this sub-section, the strain paths in response to stress-controlled uniaxial loading along the RD and TD are presented. All 72 strain paths for 6 different initial texture, 6 dislocation-based hardening models and 2 uniaxial loading paths are shown in Fig. 4.

The degree of uniaxiality between the stressing (or straining) and the resulting strain (or stress) condition is reflected in the r-value (Lankford parameter). The r-values during uniaxial loading in the RD and TD were additionally calculated by the Lankford subroutine built into VPSC 7b and are given in Table 5. Along the RD, the applied uniaxial stress results in a uniaxial strain response for all considered texture components (except for the Brass component). The Brass component (the fifth row of Fig. 4) causes the strain path to be slightly off the RD and moving towards the PS-RD strain condition, in particular when the COL latent hardening is active. Along the TD, applied uniaxial stress also leads to a uniaxial strain response in this direction for the initial Random, Cube and Brass, but not for Copper, S and Goss components. The dependence of the resulting strain paths during uniaxial stressing on the latent hardening sources is much weaker than that solely associated with texture (the $2^{\text {nd }}-6^{\text {th }}$ columns versus the $1^{\text {st }}$ column in Fig. 4).

Table 5: r-values for different textures during uniaxial tension parallel to the RD and TD 


\begin{tabular}{lcccccc}
\hline Texture & Random & Cube & Copper & $\mathrm{S}$ & Brass & Goss \\
\hline Tension // RD & 0.9 & 1.09 & 1.03 & 1 & 0.59 & 1.01 \\
\hline Tension // TD & 0.81 & 1.09 & 0.03 & 0.27 & 0.98 & 87.34 \\
\hline
\end{tabular}

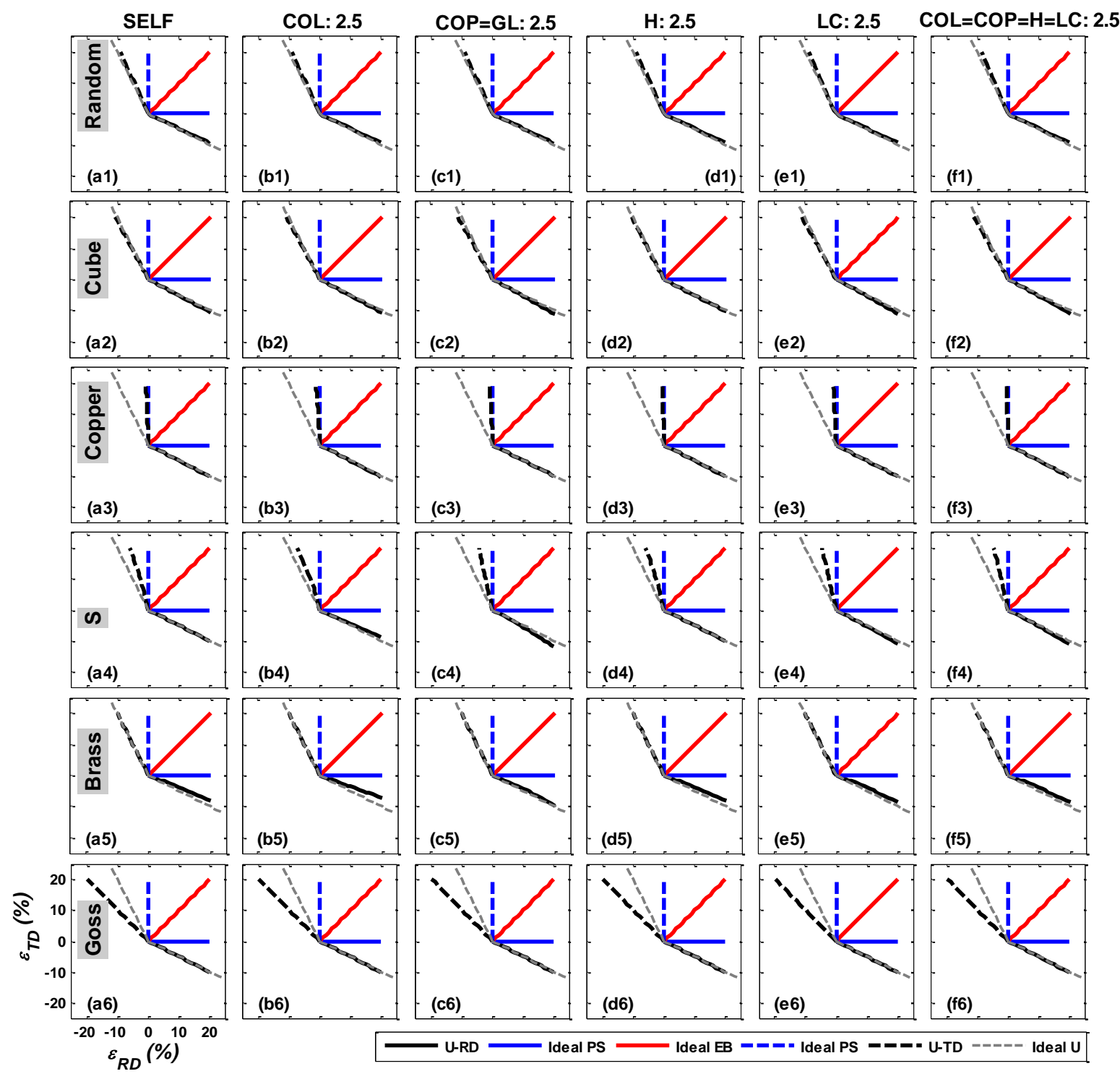

Figure 4: Strain paths in response to uniaxial stressing along the RD and TD directions. Grey dashed lines represent the ideal strain paths during U-RD, PS-RD, EB, PS-TD and U-TD. 


\section{III.2. Plastic anisotropy during multi-axial loading: General}

All 180 stress evolution paths for 6 different initial textures, 6 dislocation-based hardening models and 5 strain paths are given in Figure 5. Guide (grey) lines show equi-biaxial $\sigma_{T D}=\sigma_{R D}$ stresses, as well as stresses $\left(\sigma_{T D}=\sigma_{R D} / 2\right.$ and $\left.\sigma_{T D}=2 \sigma_{R D}\right)$ corresponding to von Mises isotropic response stresses during EB and PS-RD and PS-TD straining. The deviations and the asymmetry parameters (referred to Fig. 3a) are shown in Fig. 6, while the parameter, $\Delta$, quantifying anisotropy in hardening rate (Fig. 3b) is given in Fig. 7. 


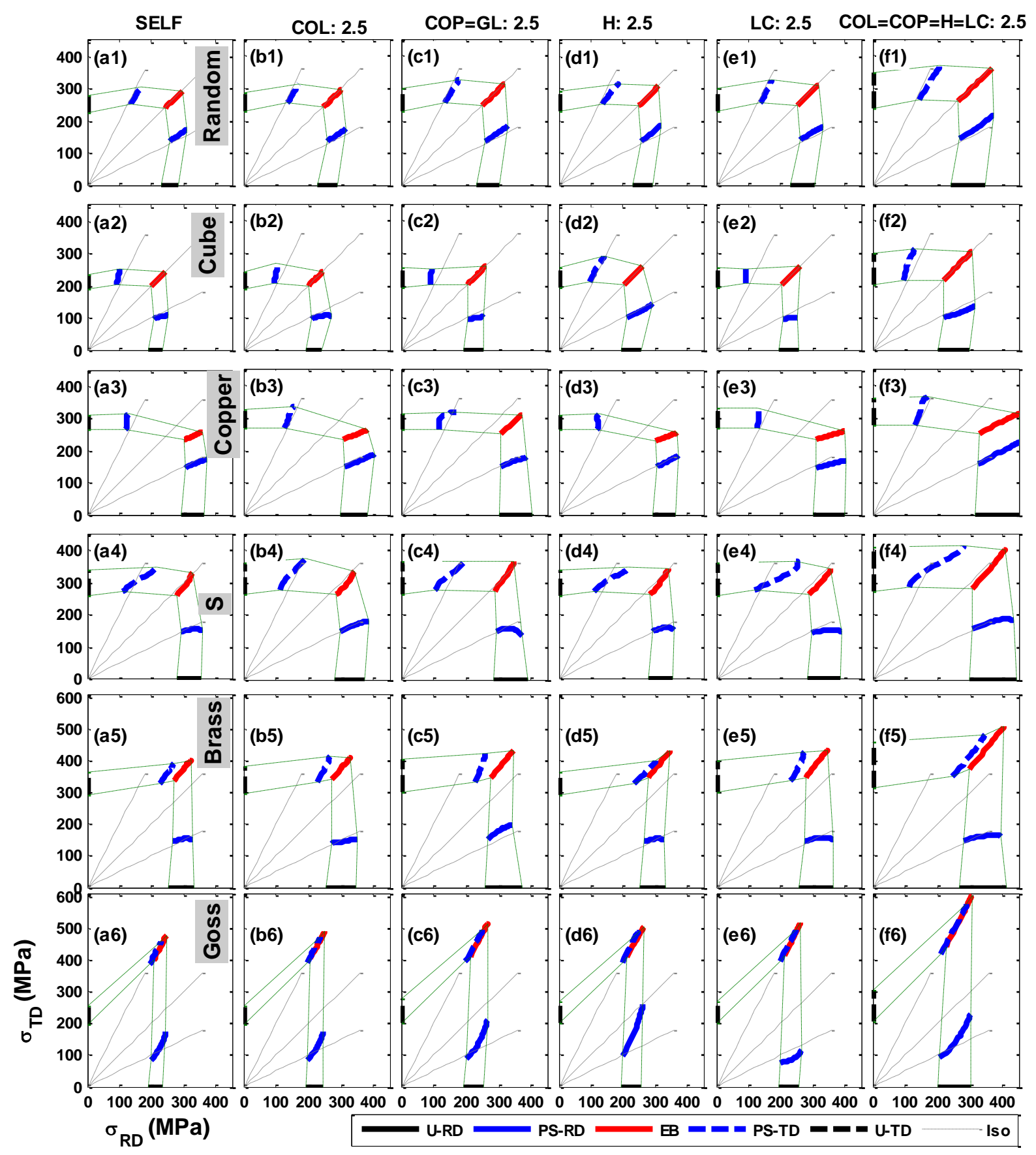

Figure 5: Stress paths in the $\left(\sigma_{T D}, \sigma_{R D}\right)$ coordinates during five linear straining paths (Table 3 ) for various initial texture conditions (rows) and hardening interaction models (columns). Note: (1) Copper texture causes the EB stress path to rotate clockwise from the isotropic EB stress line, while Brass and Goss make it rotate counter-clockwise; (2): $S$ texture leads to the most evolved stress paths amongst all considered texture; (3) the envelopes (green dashed lines) consist of stresses at plastic work values that are equal to those at strains of 1 and $20 \%$ during U-RD. 


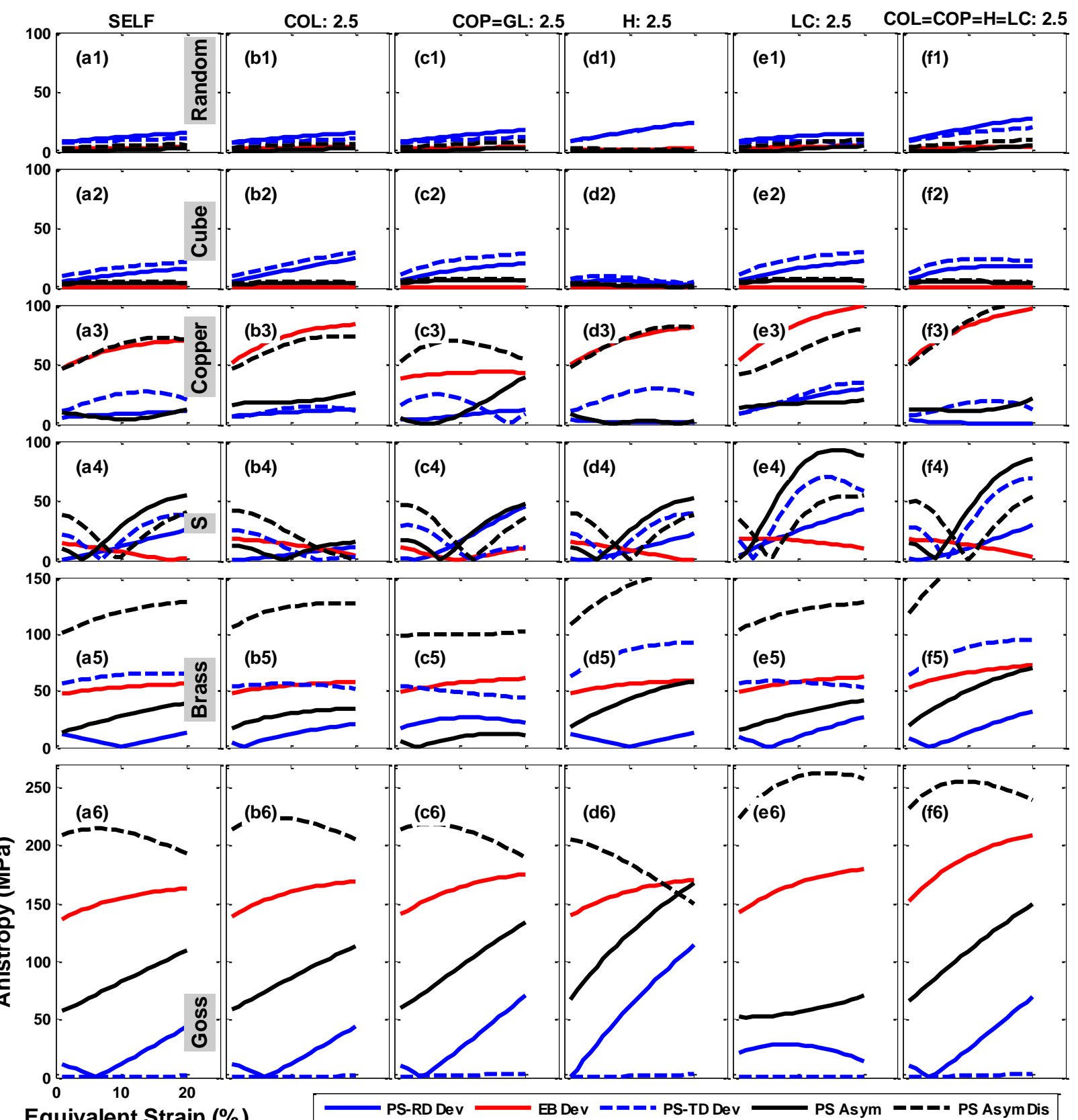

Equivalent Strain (\%)

Figure 6: The anisotropy of PS-RD, TD and EB stress paths in terms of their deviations from corresponding von Mises isotropic stress paths and the asymmetry of the PS-11 and PS-22 stress paths (four parameters in Fig. 3a). Note that the Goss and Brass components exhibit the largest deviations overall, but the $S$ texture results in the most evolved anisotropy. 


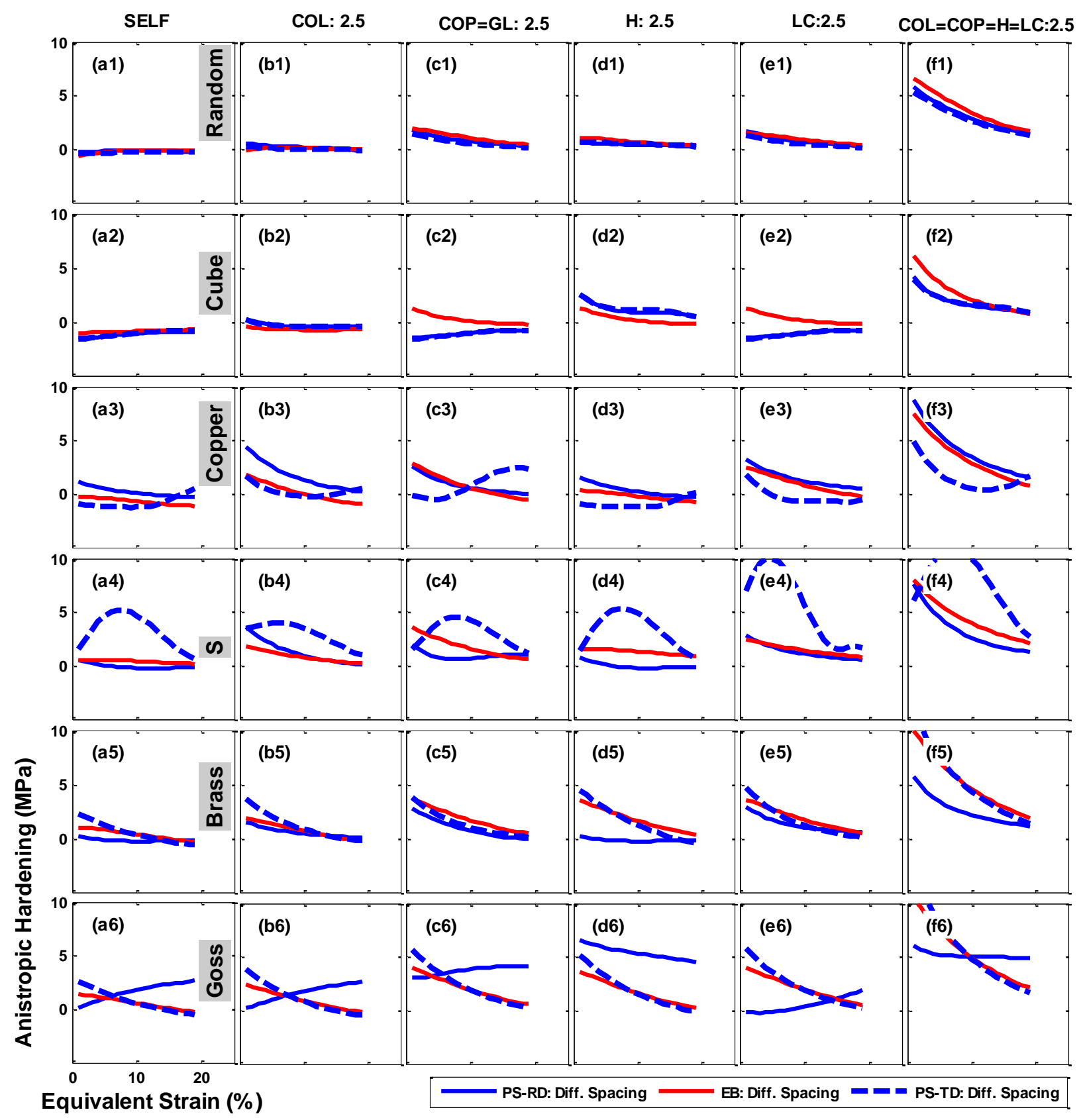

Figure 7: The anisotropic hardening parameter ( $\Delta$, Figure 3), i.e., the difference in stress increment between texture-associated stresses and von Mises stresses along a specific path (see Fig. 3b).

The initial and evolved textures associated with the self-hardening after $20 \%$ equivalent strain are shown in Fig. 8. The differences in evolved texture associated different hardening interaction models compared to initial texture are noticeable, but small. Therefore, texture 
evolution from all the different initial textures after equivalent strain of $20 \%$ by the LC latent hardening model is shown in Fig. 9 (appendix) as an example.

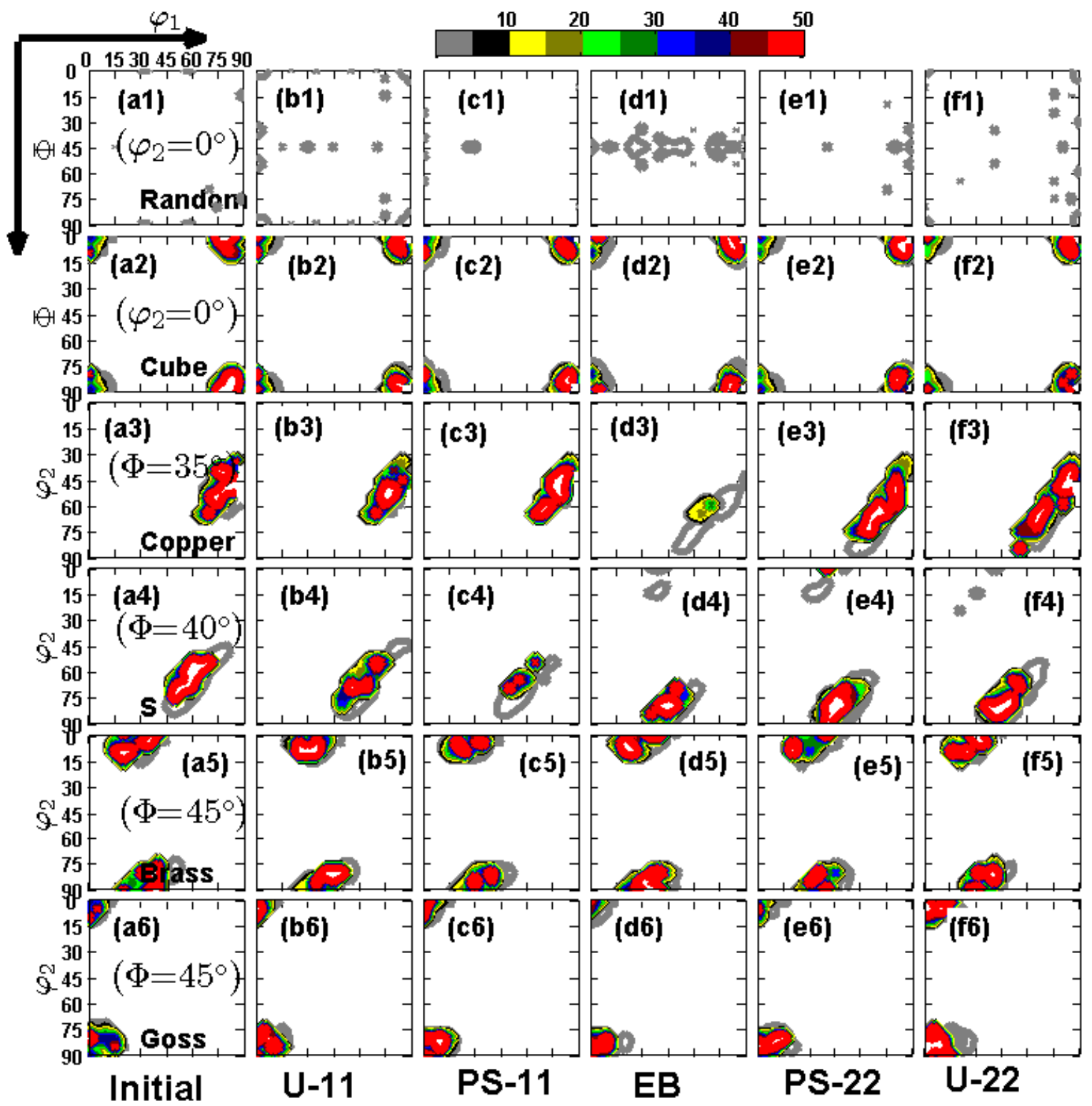

Figure 8: Initial texture (left row) compared to evolved texture after equivalent strain of $20 \%$ using the self-hardening for the five different loading paths (consecutive columns) listed at the base of the figure. Note: (1) in each row, a different initial texture component and an associated section through Bunge Euler space is shown; (2) the most texture evolution is seen for the initial S texture. 


\section{3. Random texture with self-hardening}

It is expected that if only self-hardening is active, materials with random texture should exhibit isotropic behavior because the $20 \%$ strain does not result in significant texture development. Figure $5 \mathrm{a} 1$ shows the resultant flow stresses for the 5 strain paths for the case of the initial random texture and self-hardening. The imposed equi-biaxial strains result in equi-biaxial stresses (i.e. the stress path in response to EB straining is identical to the diagonal line, Fig. 5a1). Stress paths corresponding to PS-RD and PS-TD are very close to von Mises isotropic stress lines, and are fit well by $\sigma_{T D}=(1 / 1.9) \sigma_{R D}$ and by $\sigma_{T D}=1.9 \sigma_{R D}$, respectively. The EB deviation and PS asymmetrical parameters are close to zero, while the deviations of the resulting stress paths during PS-RD and TD are negligible (Fig. 6a1). Similarly, the anisotropic hardening parameter for all stress paths is essentially zero (Fig. 7a1). Consequently, the behavior of materials with random initial texture under the influence of self-hardening is close to isotropy in the beginning of deformation, as expected. However, upon further loading, there are some noticeable deviations from isotropic response, shown in Fig. 6a1.

To see if the noticeable deviations result from the texture evolution, Figs. 8a1-f1 show the comparison of the initial Random texture and evolved texture after the equivalent strain of $20 \%$ along the five different strain paths. The initial uniform texture is slightly biased toward the $\phi_{1}=90^{\circ}$ line, which is likely an effect of the finite number of grains and/or a bias error in MTEX. Uniaxial strains along the RD result in weak texture that consists of Cube, Brass and Goss components, Fig. 8b1. Plane strain along the RD results in a mix of Goss and Brass components, Fig. 8c1. EB strains result in a fiber texture running between Goss and Brass and their mirrors near $\phi_{1}=90^{\circ}$, Fig. 8d1. PS-TD and U-TD appear to be mirrors of the results for the PS-RD and U-RD, Figs. $8 \mathrm{e} 1$ and $8 \mathrm{f1}$, but with slightly higher intensities perhaps because of the bias toward the TD as observed in the initial texture.

\section{III.4. Random texture with latent hardening}

To study the influence of dislocation-based latent hardening on the plastic anisotropy in the absence of preferred texture (i.e., random), the same random initial texture with one of four latent hardening sources was used in five sets of VPSC simulations. In each of the first four simulation sets, the same value of 2.5 was assigned to latent hardening coefficients corresponding to either the $\mathrm{COL}, \mathrm{COP}=\mathrm{GL}, \mathrm{H}$ or LC type of dislocation interactions, Table 2. The results of these four sets of VPSC runs are shown in Figure $5 b 1$ to e1. The self-hardening simulation set (given in Figure 5a1 and in Sect. III.3) is used as a reference to compare with 
latent hardening models. In the last simulation set, $\mathrm{COL}=\mathrm{COP}=\mathrm{GL}=\mathrm{H}=\mathrm{LC}=2.5$, shown in Figure $5 f 1$, all latent hardening types were active and assigned the same strength of 2.5 times stronger than the self-interaction.

Figs. 5a1-e1 show there are only small differences between the different hardening models when the initial texture is random. The flow stresses show some differences in the proportional $\sigma_{\mathrm{RD}}$ and $\sigma_{\mathrm{TD}}$ stresses, manifested as small curvature changes in the PS stresses in case of the $\mathrm{H}$ interactions. The $\mathrm{COL}=\mathrm{COP}=\mathrm{GL}=\mathrm{H}=\mathrm{LC}=2.5$ results in larger flow stresses than any single dislocation interaction type. The largest flow stresses are in agreement with the highest anisotropic hardening rates seen in the $\mathrm{COL}=\mathrm{COP}=\mathrm{GL}=\mathrm{H}=\mathrm{LC}=2.5$, Fig. $7 \mathrm{f} 1$. Concerning the plastic anisotropy, as shown in Fig. 6b1-f1, the latent hardening leads to more deviations of PSRD and PS-TD stress paths compared to those of the self-interaction (in particular the $H$ ). However, the EB deviation and PS-asymmetry are very close to zero. As mentioned previously, the differences in evolved texture were small when comparing different hardening interaction models.

\section{III.5. Initial texture: Cube}

For an initially Cube-oriented material and self-hardening, Fig. 5a2, the flow stresses are somewhat similar to those of uniform orientations. Overall, the flow stresses are less than those calculated for the uniform texture, at equivalent strains of $1 \%$ and at $20 \%$. Notably, the stress paths during plane strain of initially Cube-texture materials diverge from von Mises isotropic stress line toward the RD (or TD) axis in case of PS-RD (or PS-TD), Fig. $5 a 2$.

Including latent hardening does not induce any significant plastic anisotropy during EB straining as shown in Figs. 5b2-f2, Figs. 6a2-f2 and Fig. 7a2-f2. In addition, the stress paths for PS-RD and PS-TD are almost symmetric about the equi-biaxial stress line for all hardening types (the PS Asym and PS AsymDis in Fig. 6a2-f2 are close to zero). In general, the latent hardening models (except for the $\mathrm{H}$ type) slightly strengthen the anisotropy in terms of the deviations of the plane-strain stress paths (PS-RD and PS-TD deviations in Fig. 6 b2-f2) from the corresponding von Mises isotropic lines. Moreover, $\mathrm{COP}=\mathrm{GL}$ and $\mathrm{LC}$ latent hardening models result in much less expansion in PS flow stresses compared to stresses during uniaxial and equi-axial straining, leading to a wasp-waisted iso-work curve, Figs. $5 \mathrm{c} 2$, and $5 \mathrm{e} 2$. Both the $\mathrm{COL}$ and $\mathrm{H}$ latent hardening models result in a larger expansion of the PS stresses than the $C O P=G L$ and LC. Concerning the plastic anisotropy under the presence of the Cube texture, the $\mathrm{H}$ latent hardening causes the least anisotropy after equivalent strain of $20 \%$, Fig. 6d2, amongst all hardening sources including the self-hardening. In the case where all latent hardening 
interactions are active and have the same strength, the shape of stress envelope looks similar to (but larger than) that of self-hardening. Concerning the texture evolution, the Cube texture remains almost unchanged under all strain paths under the presence of the self-hardening as shown in Fig. 8a2-f2. A similar result occurred for other latent hardening models, for example when LC hardening is active, Fig. 9.

\section{III.6. Initial texture: Copper}

The Copper component causes much more anisotropy compared to those associated with Cube and Random. For Copper-oriented initial texture and self-hardening, the flow stress response during $\mathrm{EB}$ straining starts at a higher stress in the RD and continues to evolve in such a way that $\sigma_{R D}$ is higher than $\sigma_{T D}$ Fig. 5a3. Upon further EB loading, the EB flow stress even diverges more from the von Mises isotropic stress line, indicating a higher hardening rate in the RD than the TD. Although the PS-RD flow stress follows the corresponding isotropic line, the PS-TD flow stresses start near the isotropic value then evolve such that the $\sigma_{R D}$ stress is almost constant.

The PS-RD stress path approximately follows the isotropic stress values irrespective of the types of dislocation interactions. However, all latent hardening sources (except for the $\mathrm{COP}=\mathrm{GL}$ ) increase the plastic anisotropy compared to self-hardening (Figs. 5b3-f3 versus Fig. $5 \mathrm{a} 3$ and Figs. 6b3-f3 versus Fig. 6a3). Amongst all individual latent hardening sources, the LC results in the largest $E B$ (Fig. $6 \mathrm{~b} 3$ ), while the $C O P=G L$ latent hardening causes the least $E B$ deviation, Fig. $6 \mathrm{c} 3$. In addition, $\mathrm{COP}=\mathrm{GL}$ results in a significant curvature change in the PS-TD stress path back toward the corresponding PS-TD isotropic line, Fig. $5 \mathrm{c} 3$, leading to the least PS-TD deviation at large strains, Fig. 6c3.

The texture evolution shown in Fig. 8a3-f3 shows that the initial Copper texture under U-RD and PS-RD strain paths is rather stable although there was a reduction in intensity. However, the EB, PS-TD and U-TD strain paths cause rotation of grains toward the $S$ orientation to form part of the $B$ fiber. Latent hardening might slightly enhance the trend of texture development from Copper to S, in particular for the LC latent hardening (Fig. 9d3-f3 versus Fig. 8d3-f3).

\section{III.7. Initial texture: S}

The initial $S$ texture results in the most interesting evolution in plastic anisotropy and in the hardening variation, Figs. 5a4-f4, 6a4-f4 and 7a4-f4. Stress paths for an initial S texture start out quite similar to that of the Copper component, but later evolve to be more like Brass (shown later in Sect. III.8). For example, the EB stress path is somewhat similar to that of the Copper 
texture, with $\sigma_{T D}<\sigma_{R D}$, for low EB strain values, Fig. 5a4-f4. However at larger strains. e.g., higher than about $10 \%$ for the $\mathrm{COP}=\mathrm{GL}$, Fig. 5c4, the stress path changes direction, crosses the isotropic equi-stress line, and ends with $\sigma_{T D}>\sigma_{R D}$. Similarly, the PS-TD stress path begins on the left side of the isotropic PS-TD stress line, but rapidly crosses the isotropic line to get closer to the equi-biaxial stress. The PS-TD stress path of the Brass texture is also seen to be in between the isotropic PS-TD and isotropic EB stress lines, Fig. 5a5-f5. The LC latent hardening model even causes a second inflection point of the PS-TD flow stress to make the stress path, Fig. 5e4, eventually curved similar to the PS-TD stress path of the Brass texture at large strains, Fig. 5e5. In addition, the PS-RD stress paths ( $4^{\text {th }}$ row of Fig. 5 ) appear to follow the isotropic line for strain $<3 \%$ similar to the PS-RD stress paths of Copper texture ( $3^{\text {rd }}$ row of Fig. 5 ). However, for strain $>10 \%$, they curve toward the RD axis similar to PS-RD stress lines of the Brass texture $\left(5^{\text {th }}\right.$ row of Fig. 5$)$.

Among all the hardening models (including the self-hardening), the COL leads to the least plastic anisotropy, Fig. 6b4, while the LC causes the most anisotropy, Fig. 6e4. The PS-TD hardening rate shows the most variation from the isotropy compared to the other loading paths, Fig. 7a4-f4. Amongst all the latent hardening models, LC causes the largest difference in the PS-TD hardening rate from the isotropy, Fig. 7b4-f4. Owing to the strongly evolving PS-TD stress, the PS asymmetry and the PS-TD deviation are dominant in the plastic anisotropy associated with this texture, while the EB deviation generally diminishes, Figs. 6a4-f4.

The texture evolution of initial $S$ texture shows the most rapid changes amongst the all considered textures, Figs. 8a4-f4. The U-RD strain path diminishes the $S$ texture intensity and shifts the orientation toward the Brass component. The PS-RD strain path appears to enhance these changes. EB, PS-TD and U-TD strain paths cause the most obvious changes in the orientation and intensity to rotate toward the Brass component.

\section{III.8. Brass component}

An initial Brass texture results in $\sigma_{T D}>\sigma_{R D}$ during EB straining and significant EB and PS-TD deviations, Figs. 5a5-f5 and 6a5-f5. For all latent hardening interactions, the EB and PS-TD stress paths rotate (but in opposite directions) from their corresponding von Mises stress lines by an almost same angle, Figs. 5a5-f5. The two stress paths are close to the other, and be in the middle between the isotropic EB and PS-TD stress lines, Figs. 5a5-f5. The PS-RD stress paths are quite close to the corresponding isotropic line, but curve slightly toward the RD axis. Amongst all considered individual latent hardening types, Fig. $6 \mathrm{~d} 5$ shows that the $\mathrm{H}$ causes the 
most severe plastic anisotropy due to increasing PS-TD deviation and PS-asymmetry. The H latent hardening causes the PS-TD and EB stress paths to be very close to each other, Fig. $5 d 5$. Although there are some changes in the intensity of anisotropic hardening rates due to different individual dislocation interactions (Fig. 7a5-f5), the rates evolve almost in a same manner. The initial Brass texture changes only slightly in intensity and its locations are essentially unchanged, Figs. 8a5-f5 and 9a5-f5.

\section{III.9. Initial texture: Goss}

The Goss texture causes the most severe anisotropy during EB straining of all of the initial textures explored. The stress path of initially Goss texture during EB straining gets very close to that during PS-TD and they both follow the isotropic PS-TD stress path, Fig. 5a6-f6. While the stress paths in response to PS-TD and EB follow the same behavior irrespective of hardening sources, latent hardening leads to significant changes in the stresses under PS-RD. The stress path curves more toward the equi-stress line, Fig. 5a6-f6. The $\mathrm{H}$ interaction dramatically causes the PS-RD stress path evolving curvature, resulting in the equi-biaxial stress at PS-RD of $20 \%$, Fig. 5d6. Fig. 6a5-f5 shows the EB deviation contributes most to the plastic anisotropy for this texture irrespective of the hardening sources. However, the increasing PS-RD deviation makes the materials behave more anisotropically. Similar to the initial Brass texture, most of the changes in texture development from the initial Goss texture are seen in its intensity, Figs. 8a6$\mathrm{f} 6$ and 9a6-f6.

\section{Discussion}

\section{IV.1. Influences of initial texture and latent hardening on strain paths during uniaxial loading}

The uniaxial loading in the RD (or TD) direction was done by imposing both velocity gradient in the RD (or TD) direction and the normal stress components of in the TD and ND (or RD and ND) directions (to assure that $\sigma_{T D}=\sigma_{N D}=0$ or $\sigma_{R D}=\sigma_{N D}=0$ for uniaxial loading in the RD or TD, respectively). This imposed loading condition is different to those of PS and EB straining during which the velocity gradient were controlled. Because all considered textures (except the Brass) have an r-value in the RD equal (or close) to unity (Table 5), the imposed loading condition in the RD results in the ideal isotropic strain path regardless of initial texture (except for the Brass texture) or hardening model, Fig. 4. The exception for the Brass texture is because the r-value of this texture in the RD is smaller than 1 (see Table 5). 
The similar imposition of uniaxial loading along the TD also results in uniaxial straining for the initial Random, Cube and Brass textures, probably because the r-values along the TD of these initial textures are quite close to 1 . However, uniaxial straining is not observed for the initial Copper, $S$ and Goss textures, Fig. 4 because the r-values of these textures during U-TD are significantly different from 1 (Table 5). The r-values in the TD of Copper and S textures are significantly smaller than 1 , and that explains why the U-TD strain path goes toward the plane strain condition. In contrast, the r-value of the Goss texture during tension in the TD is very high, leading the resultant strain path to be toward the simple shear strain condition. All latent hardening sources have little influence on the strain paths compared to the self-hardening. Consequently, the strain paths under uniaxial loading conditions are mainly governed by the initial textures and only weakly by hardening types.

\section{IV.2. Role of initial texture in plastic anisotropy of FCC materials during multi-axial straining}

The response of the initial Random texture under the presence of only self-hardening is quite close to the von Mises isotropic behavior, although small deviations of response stress and strain paths from the ideal von Mises isotropic lines are seen for this texture, Figs. 5a1, 6a1 and 7a1. These slight deviations probably occur because only 2,000 grains were considered. Increasing the number of grains can make the materials behave more isotropically. Concerning the degree of anisotropy, compared to the von Mises isotropic behavior the other considered textures (not including the Random texture) without latent hardening, the initial Goss causes the most severely anisotropic response, Figs. $5 \mathrm{a} 6$ and $6 \mathrm{a} 6$, while the Cube leads to the least, Figs. $5 \mathrm{a} 2$ and $6 \mathrm{a} 2$.

Different stresses are initially required along each loading direction to activate slip in the specifically oriented grains. In order to explain the texture-induced anisotropy (in particular the initial yielding), stresses required to move dislocations in twelve slip systems when loading in either the RD or TD were calculated for all considered orientations, and are given in Table 6. Corresponding values of the Schmid factor of each slip system for loading along RD (or TD) are given in Table 7 in the Appendix. Table 6 shows that it requires $198 \mathrm{MPa}$ to initiate slip in Gossoriented grains during loading in the RD and TD. However, at the same required stress, the number of active slip systems in the TD direction is half of that in the RD. During EB, the same amount of strain along the RD and TD is required. Therefore the stress that is required to accommodate the same strain amount in the TD should be double that in the RD. This explains why the $\sigma_{T D}$ is seen to be twice of the $\sigma_{R D}$ during EB for the initial Goss texture, Fig. 5a6. 
Similarly, Backofen [49] showed that the vertex of the yield surface corresponding to the EB straining of a Goss-oriented FCC single crystal aligns to the line where $\sigma_{T D}=2 \sigma_{R D}$.

During PS-RD straining, (1) the material experiences tensile loading along the TD and (2) it requires the intensity of slip activity along the TD to be half of that along $R D$ in order to maintain the plane strain constraint, i.e., the principal minor strain $\varepsilon_{T D}=0$. If the required stress (198 $\mathrm{MPa}$ ) and the number of active slip systems are the same when loading in the RD and TD, the PS-RD stress path should follow the relationship $\sigma_{T D}=\sigma_{R D} / 2$ as expected for the isotropic behavior. However, because of the number of active slip systems when loading along the TD is half that along the RD (the last two rows in Tables 6 and 7), required stress along the TD should be higher than $\sigma_{R D} / 2$. Therefore, the PS-RD stress path deviates from the $\sigma_{T D}=\sigma_{R D} / 2$ line and curves upward, Fig. 5a6. By contrast, the number of active slip systems during loading along the RD is double of those along the TD as shown in the last two rows in Tables 6 and 7. Consequently, $\sigma_{T D}=2 \sigma_{R D}$ during PS-TD.

Table 6: Initially required stresses to activate dislocation movement in different slip systems of oriented grains when loading along the RD (or TD). Note: 1. $\tau_{0}=81 \mathrm{MPa}, 2$. Required stresses larger than the calculated stresses shown in Fig. 5 are not of interest and are represented by “-".

\begin{tabular}{|c|c|c|c|c|c|c|c|c|c|c|c|c|c|}
\hline Texture & & 1 & 2 & 3 & 4 & 5 & 6 & 7 & 8 & 9 & 10 & 11 & 12 \\
\hline \multirow{2}{*}{ Cube } & $\mathrm{RD}$ & -- & 198 & 198 & -- & 198 & 198 & -- & 198 & 198 & -- & 198 & 198 \\
\hline & TD & 198 & -- & 198 & 198 & -- & 198 & 198 & -- & 198 & 198 & -- & 198 \\
\hline \multirow{2}{*}{ Copper } & $\mathrm{RD}$ & -- & 290 & 290 & 300 & 300 & -- & -- & -- & -- & 300 & -- & 300 \\
\hline & TD & 200 & -- & 200 & 200 & -- & 190 & -- & -- & -- & -- & -- & -- \\
\hline \multirow{2}{*}{$S$} & $\mathrm{RD}$ & -- & -- & -- & 254 & 212 & -- & -- & -- & -- & 317 & -- & 248 \\
\hline & TD & 268 & -- & 322 & 184 & -- & 199 & -- & -- & -- & -- & -- & -- \\
\hline \multirow{2}{*}{ Brass } & $\mathrm{RD}$ & -- & -- & -- & 299 & 198 & -- & -- & 294 & 294 & 299 & -- & 198 \\
\hline & TD & -- & -- & -- & 299 & -- & 300 & -- & 294 & 294 & 299 & 299 & -- \\
\hline \multirow{2}{*}{ Goss } & $\mathrm{RD}$ & -- & 198 & 198 & -- & 198 & 198 & -- & 198 & 198 & -- & 198 & 198 \\
\hline & TD & -- & 198 & 198 & -- & -- & -- & -- & 198 & 198 & -- & -- & -- \\
\hline
\end{tabular}


Compared to the initially required stresses (198 MPa) for Goss-oriented grains, higher stresses (198, 294 and $299 \mathrm{MPa}$ ) are required for Brass-oriented grains to activate a sufficient number of slips (at least 5 slip systems) to accommodate plastic strain when loading in the RD. The required stresses in the $\mathrm{RD}$ to activate slips are lower than those in the TD (Table 6). Consequently, the higher required stresses in the TD direction explain the larger expansion of the U-TD stress of Brass, Fig. $5 a 5$ vs. Fig. 5a6. In addition, because required stresses in TD are generally larger than those in the $\mathrm{RD}$, the Brass texture induces plastic anisotropy $\left(\sigma_{T D}>\sigma_{R D}\right)$ during EB, similar but to a lesser extent compared to the Goss, Fig. $5 a 5$ vs. Fig. 5a6. By contrast, the Copper texture causes the required stresses to activate at least five slip systems along the RD (290 and $300 \mathrm{MPa}$ ) to be smaller than those along the TD (190 and $200 \mathrm{MPa}$ ). Moreover, the ratios of required stresses in the RD to those in the TD are equal (or close) to 3/2. As a result, the Copper results in the stress path in response of EB to be close to $\sigma_{R D}=3 \sigma_{T D} / 2$, similar to what is seen for Copper-oriented FCC single crystals during EB [49].

To a certain extent, Goss and Copper are quite similar in the sense that they cause two extremes of plastic anisotropy amongst all considered textures. In detail,

(1) While the Goss causes EB stress paths deviate most from the isotropic EB line toward $\sigma_{T D}$, the Copper texture causes the largest EB deviation toward the $\sigma_{R D}$ axis away from the isotropic stress line.

(2) During stress-controlled uniaxial loading, Goss takes the TD strain path toward simple shear, while Copper takes it towards plane strain, Figs. 4a3 and 4a6.

(3) The Goss does not affect the PS-TD stress path deviation, Fig. 5a6, while the Copper causes very little PS-RD deviation, Fig. 5 a3.

Although Goss and Copper appear to cause two extremes of plastic anisotropy as mentioned above, the most complex plastic anisotropy is seen for the $S$ texture (Sect. III.7). During in-plane straining, i.e., PS-RD, EB and PS-TD, the $S$ results in anisotropy that has intermediate characteristics in between that of Copper and Brass, Figs. 5a4 and 5a5. The S is intermediate texture between Brass and Copper (but is closer to the Copper position, Fig. 2). Therefore, the $S$ texture initially results in almost the same anisotropy as that of Copper, but to a much smaller extent, Fig. $5 a 4$ vs. Fig. 5a3. After substantial deformation, initially S-oriented materials exhibit anisotropic behavior similar to initially Brass-textured materials because the initial $S$ texture develops into Brass to form a $\beta$-fiber, Figs. 8a4-f4. 


\section{IV.3. Roles of latent hardening in plastic anisotropy of Random texture}

It is interesting to note that the role of the latent hardening on plastic anisotropy is not significant in the case of no preferred crystallographic texture. The plastic anisotropy of Random texture is nearly the same irrespective of latent hardening sources, Fig. 5b1-f1. One of the reasons why the material still behaves isotropically even with latent hardening is probably that the random texture allows faster hardening slip systems to be active, which are compensated by slower hardening slip systems or vice versa. Consequently, averaging a large number of uniformly oriented grains can result in isotropic response.

Another reason is related to the development of texture. Figs. 8a1-f1 show an example of how the initially random texture evolves during loading for the self-hardening model. In general, the texture evolution is the same for all of the latent hardening models, except the intensity of texture might be higher, e.g., Fig. 9a1-f1 vs. 8a1-f1. All hardening models predict the same development during EB straining from the initially uniform texture into $\alpha$-fibers consisting of variants of Goss and Brass components, e.g., Goss $(0,45,0)$, Brass $(35,45,0)$, Brass $(55,45,0)$ and Goss $(90,45,0)$, Figs. $8 \mathrm{~d} 1$ and $9 \mathrm{~d} 1$. Recall that in Bunge Euler space, $\left(\varphi_{1}=0\right)$ is the rolling direction and $\left(\varphi_{1}=90\right)$ is the transverse direction. The Brass and Goss components close to the $\left(\varphi_{1}=90\right)$-plane compensate for the anisotropic response induced by Goss $(0,45,0)$ and Brass $(35,45,0)$ textures close to the $\left(\varphi_{1}=0\right)$-plane, resulting in no EB Dev, Fig. 6b1-f1. During plane straining, the initially random texture develops weak texture comprising Goss and

Brass without their counterparts near the $\left(\varphi_{1}=90\right)$-plane for PS-RD (or the $\left(\varphi_{1}=0\right)$-plane for PS-TD), Figs. 8c1, 8e1 9c1 and 9e1. Consequently, there is some minor plastic anisotropy (see the PS-RD and PS-TD deviations Figs. 6b1-f1).

\section{IV.4. Synergistic roles of texture and latent hardening on plastic anisotropy}

The presence of both texture and latent hardening results in more complex anisotropy during multi-axial loading. The influence of latent hardening on anisotropy depends on texture or vice versa. For instance, latent hardening causes significant plastic anisotropy only when there is presence of preferred texture, in particular Copper, S, Brass and Goss. Similarly, the textureinduced plastic anisotropy is changed under the presence of an active latent hardening. For example, comparing to the self-hardening, the $\mathrm{COL}$ latent hardening significantly reduces plastic anisotropy for $S$ texture, Fig. $6 \mathrm{~b} 4$, leading to the least plastic anisotropy amongst all latent 
hardening sources. However, the COL does not cause much difference for other textures. Similarly, the $\mathrm{H}$ latent hardening causes the least plastic anisotropy for the Cube texture, but for the Brass the COL induces the most anisotropy, Fig. 5, amongst all individual hardening types (including the self-hardening). Consequently, better understanding of the synergistic roles of latent hardening and texture condition can provide more insights into the plastic anisotropy of materials.

It should be noted that for the same initial texture, different individual latent hardening types can change the texture-induced plastic anisotropy in different ways, sometimes having opposite effects. The best demonstration can be seen by looking at the evolution of the PS-TD stress path of Copper-oriented aggregate under the presence of different individual latent hardening types, Fig. 5a3-f3. While COL, H and LC tend to make the PS-TD stress paths move toward the TD axis, the $C O P=G L$ makes the PS-TD stress path curve dramatically toward the diagonal line, Figs. 5b3, d3 and e3 versus Fig. 5c3. Because different latent hardening types can have opposite effects on plastic anisotropy, a simple treatment of all non-self-hardening having the same strength can result in an approximately isotropic response. Consequently, simple superposition of all latent hardening types might not reflect the complex role of latent hardening on plastic anisotropy (the $6^{\text {th }}$ column versus the $2^{\text {nd }}$ through $5^{\text {th }}$ columns, Figs. 5 and 6 ).

Overall, amongst all considered preferred crystallographic textures and latent hardening, the least plastic anisotropy is seen under the presence of both Cube texture and $\mathrm{H}$ hardening, Fig. $6 \mathrm{~d} 2$. This is likely because:

(1) Cube texture is highly symmetric, Fig. 2. In addition, VPSC simulation also predicts that initial Cube texture is (meta-)stable during EB straining, Figs. 8d2 and 9d2.

(2) $\mathrm{H}$ interaction type results in dislocation junctions orienting in the [100]-direction that is also highly symmetric.

As referred to in Sect. IV.2, the most complex evolution of anisotropy is seen for S texture. The LC latent hardening can even enhance this complexity, Figs. $6 \mathrm{e} 4$ and 7e4. The LC latent hardening can slightly accelerate the development of the initial $S$ texture into Brass components to form a $\beta$-fiber, in particular during EB and PS-TD, Fig. 9d4-e4 vs. 8d4-e4. This enhanced texture development associated with the LC hardening is likely responsible for the majority of the evolution of plastic anisotropy, Figs. $5 \mathrm{e} 4$ and $6 \mathrm{e} 4$.

As mentioned earlier, there is still a debate as to whether latent hardening plays a significant role on the plastic anisotropy. For example, while there was an observation showing that the 
effect of latent hardening on plastic anisotropy was negligible [6] (note: authors of this study did not show the initial texture of their simulation), other observations on materials with initially $\alpha$-fiber [21] or a mixture of Brass, Cube, Copper, Goss and S [22] showed that latent hardening significantly affects both the texture development and deformation anisotropy. Our study has clearly shown that the role of latent hardening depends on the texture that is present. For example, if the initial texture is Cube, latent hardening insignificantly influences the anisotropy response and texture development. In addition, for an initial random texture, the latent hardening induces almost no plastic anisotropy including texture development. However, the latent hardening can greatly alter anisotropy when strong textures are present, in particular Copper, S, Brass and Goss. This explains why the latent hardening caused significant plastic anisotropy and texture development for texture mixtures of Brass and Goss or Brass, Cube, Copper, Goss and S [15, 22]. The negligible role of latent hardening on plastic anisotropy found in [6] is probably because the authors used the Random or Cube texture.

\section{Conclusions}

The separate and synergistic roles of crystallographic textures (Random, Cube, Copper, S, Brass and Goss) and latent hardening (Collinear, Coplanar, Glissile, Hirth and Lomer-Cottrell) on the anisotropy behavior of FCC polycrystalline materials were comprehensively studied in order to obtain insight into the complex plastic anisotropy during multi-axial loading. The main result is that texture and individual latent hardening types can counteract others to produce effective isotropy or act synergistically to maximize anisotropy, depending on which combination dominates. Concerning the separate influence of texture on plastic anisotropy, the Goss texture causes the most anisotropy. By contrast, if the Random texture is excluded, the Cube texture causes the least plastic anisotropy. Although Goss and Copper appear to cause two opposite extremes in plastic anisotropy amongst the considered textures, the most complex evolution of plastic anisotropy is seen for the S texture (Sect. III.7 and Sect. IV.2). A main reason for this interesting evolution in anisotropy relates to the texture development of the initial $S$ into Brass to form a $\beta$-fiber, Figs. 8a4-f4.

As already remarked, latent hardening can either enhance or significantly reduce the textureinduced anisotropy, depending on the initial texture. For example, Hirth-dominated latent hardening leads to the least plastic anisotropy for the Cube texture, but causes the most anisotropy for the Brass amongst all individual hardening types. In addition, for the same initial texture, an individual latent hardening source can have opposite effects on plastic anisotropy compared to other hardening latent sources. The material behavior would be more isotropic 
under a simple superimposition of latent hardening. Therefore, a treatment of all non-selfhardening as the same cannot reveal the complexity of plastic anisotropy.

\section{Acknowledgements}

We would like to thank Dr. Carlos Tomé and Dr. Ricardo Lebensohn at Los Alamos National Laboratory (USA) for providing the VPSC program. This work was supported by the National Institute of Standards and Technology (NIST) Center for Automotive Lightweighting. The financial support of Materials Genome Initiative at NIST is also acknowledged.

\section{References}

[1] Taylor GI. Plastic strain in metals. Journal of the Institute of Metals 1938;62:307.

[2] Asaro; RJ, Needleman A. OVERVIEW NO. 42: TEXTURE DEVELOPMENT AND STRAIN HARDENING IN RATE DEPENDENT POLYCRYSTALS Acta Mater 1985;33:923.

[3] Lebensohn RA, Tomé CN. A Self-Consistent Anisotropic Approach for the Simulation of PlasticDeformation and Texture Development of Polycrystals - Application to Zirconium Alloys. Acta Metall Mater 1993;41:2611.

[4] Kocks UF, Tomé CN, Wenk HR. Texture and Anisotropy: Preferred Orientations in Polycrystals and their Effect on Materials Properties. Cambridge, U.K.: Cambridge University Press, 1998.

[5] Franciosi P. The Concepts of Latent Hardening and Strain-Hardening in Metallic Single-Crystals. Acta Metallurgica 1985;33:1601.

[6] Kocks UF, Franciosi P, Kawai M. A Forest Model of Latent Hardening and Its Application to Polycrystal Deformation. Texture Microstruct 1991;14:1103.

[7] Franciosi P. Latent Hardening Effects on Deformation Texture Evolution. Jom-J Min Met Mat S 1985;37:A43.

[8] Franciosi P, Berveiller M, Zaoui A. Latent Hardening in Copper and Aluminum Single-Crystals. Acta Metallurgica 1980;28:273.

[9] Madec R, Devincre B, Kubin L, Hoc T, Rodney D. The role of collinear interaction in dislocationinduced hardening. Science 2003;301:1879.

[10] Hirth JP. On Dislocation Interactions in Fcc Lattice. Journal of Applied Physics 1961;32:700.

[11] Pham M-S, Hu L, ladicola M, Creuziger A, Rollett AD. The strain path dependence of plastic deformation response of AA5754: Experiment and modeling. AIP Conference Proceedings 2013;1567:500.

[12] Peirce D, Asaro RJ, Needleman A. Material Rate Dependence and Localized Deformation in Crystalline Solids. Acta Metallurgica 1983;31:1951.

[13] Erinosho TO, Cocks ACF, Dunne FPE. Texture, hardening and non-proportionality in BCC polycrystal deformation. Int. J. of Plasticity 2013;50:170.

[14] Erinosho TO, Cocks ACF, Dunne FPE. Coupled effects of texture, hardening and nonproportionality of strain on ductility in ferritic steel. Comp Mater Sci 2013;80:113.

[15] Miraglia M, Dawson P, Leffers T. On the influence of mechanical environment on the emergence of brass textures in FCC metals. Acta Mater 2007;55:799.

[16] Hoc T, Forest S. Polycrystal modelling of IF-Ti steel under complex loading path. Int J Plasticity 2001;17:65.

[17] Liu B, Raabe D, Roters F, Arsenlis A. Interfacial dislocation motion and interactions in singlecrystal superalloys. Acta Mater 2014;79:216. 
[18] Dequiedt JL, Denoual C, Madec R. Heterogeneous deformation in ductile FCC single crystals in biaxial stretching: the influence of slip system interactions. J Mech Phys Solids 2015 (In press).

[19] Devincre B, Kubin L, Hoc T. Physical analyses of crystal plasticity by DD simulations. Scripta Mater 2006;54:741.

[20] Pham M-S, ladicola M, Creuziger A, Hu L, Rollett AD. Thermally-activated constitutive model including dislocation interactions, aging and recovery for strain path dependence of solid solution strengthened alloys: Application to AA5754-O. Int J Plasticity 2015;75:226.

[21] Toth LS, Molinari A, Raabe D. Modeling of rolling texture development in a ferritic chromium steel. Metall Mater Trans A 1997;28:2343.

[22] Young MJ, Choi SK, Thomson PF. The Effect of Latent Hardening on the Texture of 3004 Aluminum Deformed in Plane Strain, Predicted by an Explicit Dynamic Finite Element Analysis. Journal of ASTM International 2006;3.

[23] Hu L, Rollett AD, ladicola M, Foecke T, Banovic S. Constitutive Relations for AA 5754 Based on Crystal Plasticity. Metall Mater Trans A 2012;43A:854.

[24] Tomé CN, Lebensohn RA. Manual for Code: Visco-plastic self-consistent, version 7d, 2012.

[25] Carter CB. Multiple Ribbons in Face-Centered Cubic Materials .1. Geometry and Properties of Double Ribbons. Phys Status Solidi A 1980;61:579.

[26] Frost HJ, Ashby MF. Deformation-mechanism maps the plasticity and creep of metals and ceramics. Oxford a.o.: Pergamon Press, 1982.

[27] Kocks UF. Constitutive behavior based crystal plasticity. In: Miller AK, editor. Unified constitutive equations for creep and plasticity. London etc.: Elsevier Applied Science, 1987. p.XIV.

[28] ladicola MA, Hu L, Rollett AD, Foecke T. Crystal plasticity analysis of constitutive behavior of 5754 aluminum sheet deformed along bi-linear strain paths. Int J Solids Struct 2012;49:3507.

[29] Tomé C, Canova GR, Kocks UF, Christodoulou N, Jonas JJ. The relation between macroscopic and microscopic strain hardening in F.C.C. polycrystals. Acta Metallurgica 1984;32:1637.

[30] Voce E. The relationship between stress and strain for homogeneous deformation. Journal of the Institute of Metals 1948;74:537.

[31] Kocks UF, Mecking H. A Mechanism for Static and Dynamic Recovery. In: Haasen P, Gerold V, Kostorz G, editors. Fifth Int. Conf. on the Strength of Metals and Alloys, 1979. p.345.

[32] Kocks UF. Laws for Work-Hardening and Low-Temperature Creep. J Eng Mater-T Asme 1976;98:76.

[33] Kubin L. Dislocations, mesoscale simulations and plastic flow: Oxford University Press, 2013.

[34] Chen SR, Gray GT. Constitutive behavior of tantalum and tantalum-tungsten alloys. Metall and Mat Trans A 1996;27:2994.

[35] Pokharel R, Lind J, Kanjarla AK, Lebensohn RA, Li SF, Kenesei P, Suter RM, Rollett AD. Polycrystal Plasticity: Comparison Between Grain - Scale Observations of Deformation and Simulations. Annual Review of Condensed Matter Physics 2014;5:317.

[36] Segurado J, Lebensohn RA, Llorca J, Tomé CN. Multiscale modeling of plasticity based on embedding the viscoplastic self-consistent formulation in implicit finite elements. Int J Plasticity 2012;28:124.

[37] Follansbee PS, Kocks UF. A constitutive description of the deformation of copper based on the use of the mechanical threshold stress as an internal state variable. Acta Metallurgica 1988;36:81.

[38] Kocks UF. Realistic constitutive relations for metal plasticity. Materials Science and Engineering: A 2001;317:181.

[39] Tomé CN, Necker CT, Lebensohn RA. Mechanical anisotropy and grain interaction in recrystallized aluminum. Metall and Mat Trans A 2002;33:2635.

[40] Thompson N. Dislocation Nodes in Face-Centred Cubic Lattices. Proceedings of the Physical Society of London Section B 1953;66:481. 
[41] Lomer WM. A Dislocation Reaction in the Face-Centred Cubic Lattice. Philos Mag 1951;42:1327.

[42] Cottrell A. Dislocations and plastic flow in crystals. Oxford: Clarendon Press, 1953.

[43] Cottrell AH. The Formation of Immobile Dislocations during Slip. Philos Mag 1952;43:645.

[44] Martinez E, Marian J, Arsenlis A, Victoria M, Perlado JM. Atomistically informed dislocation dynamics in fcc crystals. J Mech Phys Solids 2008;56:869.

[45] B. Devincre, T. Hoc, Kubin L. Dislocation Mean Free Paths and Strain Hardening of Crystals. Science 2008;320.

[46] Hielscher R. A MATLAB Toolbox for Quantitative Texture Analysis. Version 3.4.1, 2013.

[47] Rollett AD. Texture subroutines at http://neon.materials.cmu.edu/rollett/texture_subroutines/ accessed on October 18, 2014.

[48] Pham MS. Matlab subroutines for VPSC postprocessing. https://github.com/minhsonpham/matlab_VPSC_Wins, 2015.

[49] Backofen WA. Deformation processing. Reading, Mass.,: Addison-Wesley Pub. Co., 1972. 


\section{Appendix}

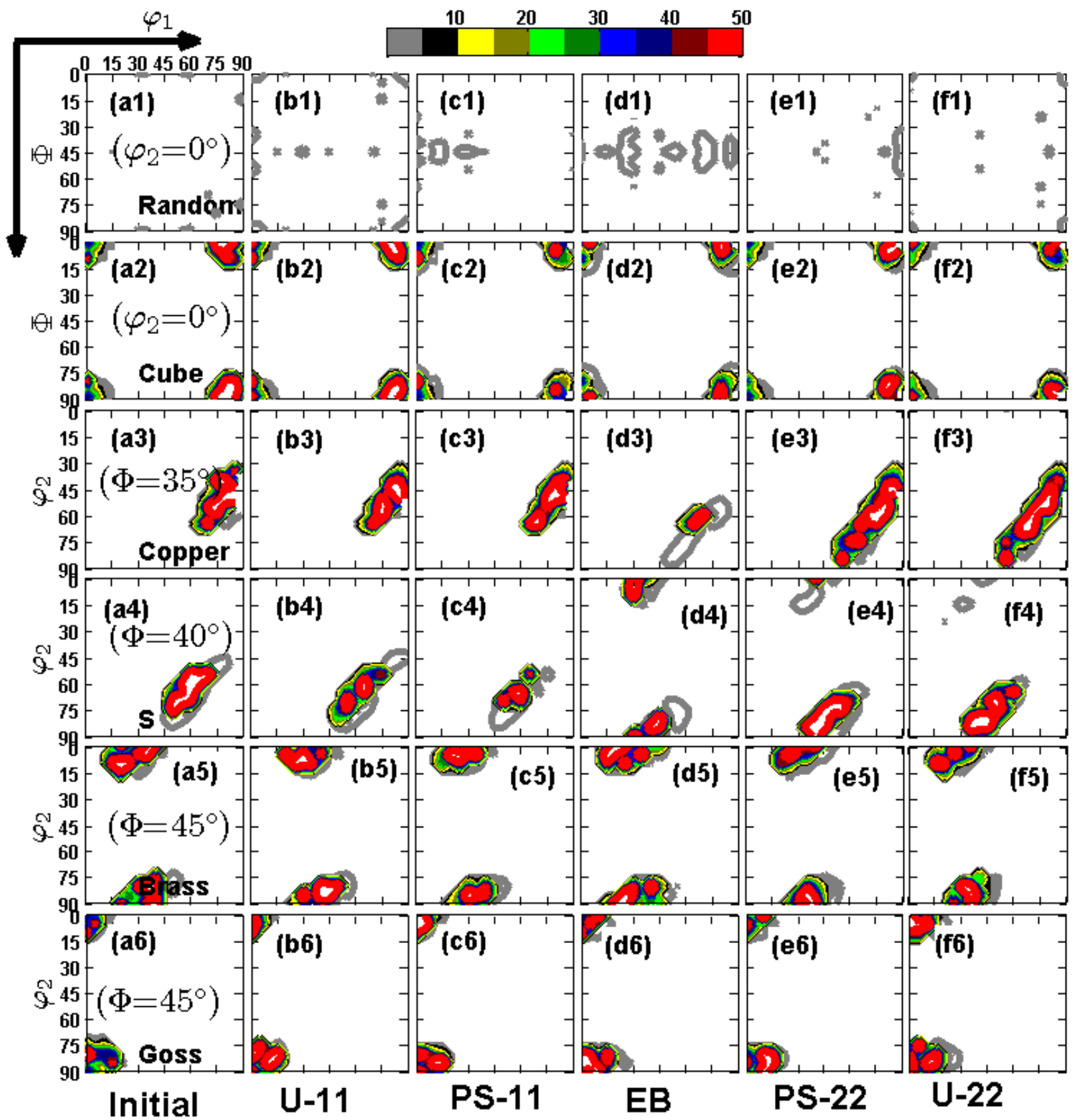

Figure 9: Initial texture (left row) and evolved texture using the LC latent hardening after strain of $20 \%$ for the five different loading paths (consecutive columns) listed at the base of the figure. Note: (1) in each row, a different initial texture component and an associated section through Bunge Euler space is shown; (2) The differences in evolved texture associated the LC hardening and self-hardening models are noticeable, but small. 
Table 7: Initially values of Schmid factor of different slip systems of oriented grains when loading direction along the RD (or TD). Note: The largest values of Schmid factor are in bold.

\begin{tabular}{|c|c|c|c|c|c|c|c|c|c|c|c|c|c|}
\hline $\mathrm{S}$ & & 1 & 2 & 3 & 4 & 5 & 6 & 7 & 8 & 9 & 10 & 11 & 12 \\
\hline \multirow{2}{*}{ Cube } & $\mathrm{RD}$ & 0.00 & 0.41 & 0.41 & 0.00 & 0.41 & 0.41 & 0.00 & 0.41 & 0.41 & 0.00 & 0.41 & 0.41 \\
\hline & TD & 0.41 & 0.00 & 0.41 & 0.41 & 0.00 & 0.41 & 0.41 & 0.00 & 0.41 & 0.41 & 0.00 & 0.41 \\
\hline \multirow{2}{*}{ Copper } & $\mathrm{RD}$ & 0.00 & 0.28 & 0.28 & 0.27 & 0.27 & 0.00 & 0.00 & 0.01 & 0.01 & 0.27 & 0.00 & 0.27 \\
\hline & TD & 0.40 & 0.02 & 0.41 & 0.40 & 0.02 & 0.42 & 0.01 & 0.01 & 0.01 & 0.01 & 0.02 & 0.01 \\
\hline \multirow{2}{*}{ S } & $\mathrm{RD}$ & 0.01 & 0.12 & 0.13 & 0.32 & 0.38 & 0.06 & 0.06 & 0.19 & 0.13 & 0.26 & 0.07 & 0.33 \\
\hline & TD & 0.30 & 0.05 & 0.25 & 0.44 & 0.03 & 0.41 & 0.06 & 0.19 & 0.13 & 0.08 & 0.10 & 0.03 \\
\hline \multirow{2}{*}{ Brass } & $\mathrm{RD}$ & 0.00 & 0.00 & 0.00 & 0.27 & 0.41 & 0.14 & 0.00 & 0.28 & 0.28 & 0.27 & 0.14 & 0.41 \\
\hline & TD & 0.00 & 0.00 & 0.00 & 0.27 & 0.00 & 0.27 & 0.00 & 0.28 & 0.28 & 0.27 & 0.27 & 0.00 \\
\hline \multirow{2}{*}{ Goss } & $\mathrm{RD}$ & 0.00 & 0.41 & 0.41 & 0.00 & 0.41 & 0.41 & 0.00 & 0.41 & 0.41 & 0.00 & 0.41 & 0.41 \\
\hline & TD & 0.00 & 0.41 & 0.41 & 0.00 & 0.00 & 0.00 & 0.00 & 0.41 & 0.41 & 0.00 & 0.00 & 0.00 \\
\hline
\end{tabular}

